\title{
INVESTIGATION OF THE DYNAMIC BEHAVIOUR OF NON-UNIFORM THICKNESS CIRCULAR PLATES RESTING ON WINKLER AND PASTERNAK FOUNDATIONS
}

\author{
Saheed Salawu ${ }^{a *}$, Gbeminiyi Sobamowo $^{b}$, Obanishola Sadiq $^{a}$ \\ ${ }^{a}$ University of Lagos, Department of Civil and Environmental Engineering, Akoka 100213, Nigeria \\ ${ }^{b}$ University of Lagos, Department of Mechanical Engineering, Akoka 100213, Nigeria \\ * corresponding author: safolu@outlook.com
}

\begin{abstract}
The study of the dynamic behaviour of non-uniform thickness circular plate resting on elastic foundations is very imperative in designing structural systems. This present research investigates the free vibration analysis of varying density and non-uniform thickness isotropic circular plates resting on Winkler and Pasternak foundations. The governing differential equation is analysed using the Galerkin method of weighted residuals. Linear and nonlinear case is considered, the surface radial and circumferential stresses are also determined. Thereafter, the accuracy and consistency of the analytical solutions obtained are ascertained by comparing the existing results available in pieces of literature and confirmed to be in a good harmony. Also, it is observed that very accurate results can be obtained with few computations. Issues relating to the singularity of circular plate governing equations are handled with ease. The analytical solutions obtained are used to determine the influence of elastic foundations, homogeneity and thickness variation on the dynamic behaviour of the circular plate, the effect of vibration on a free surface of the foundation as well as the influence of radial and circumferential stress on mode shapes of the circular plate considered. From the results, it is observed that a maximum of $8.1 \%$ percentage difference is obtained with the solutions obtained from other analytical methods. Furthermore, increasing the elastic foundation parameter increases the natural frequency. Extrema modal displacement occurs due to radial and circumferential stress. Natural frequency increases as the thickness of the circular plate increases, Conversely, a decrease in natural frequency is observed as the density varies. It is envisioned that; the present study will contribute to the existing knowledge of the classical theory of vibration.
\end{abstract}

KEYwords: Free vibration, natural frequency, Winkler and Pasternak, circular plate, Galerkin method of weighted residual.

\section{INTRODUCTION}

Application of plates is commonly found in a variety of contexts in civil, mechanical, naval, marine and aeronautic engineering. The understanding of the dynamic behaviour of the plate is germane in several contexts. For example, a plate resting on a foundation, fluid-structure interaction problems, panel flutter and plate in aeronautics, also the coupling effect of electro-magnetic and thermal fields on plate. Several engineering structures, such as railway, storage tank foundation and telecommunication mast require information on the vibration analysis of plate entrenched on foundation earlier before embarking on the design. The simplest form of modelling the mechanical behaviour of a soil foundation interaction is using the Winkler foundation. The Winkler foundation has the limitation of non-interaction between the lateral spring, thereby resulting in unreliable results. Two-parameter elastic foundations are established to cater for this interaction. The use of two-parameter elastic foundations offers a true account of the soil foundation interaction. In the study of vibration of a plate immersed in fluid, Kyeong-Hoon et al. [1] formulate a hypothetical approach for a hydroelastic analysis of a clamped edge circular plate partially in contact with a liquid. Rayleigh-Ritz method was adopted for obtaining the Eigenvalue equation. The influences of the thickness and depth of the liquid thickness on the natural frequencies were determined.

In another work, Sari and Butcher [2] proposed a numerical approach for analysing the free vibration response of an isotropic rectangular and annular Mindlin plates under damaged clamped boundary conditions using the Chebyshev collocation method. the results obtained show that the damaged boundary condition influences the natural frequency of the plate. Also, Fletcher [3] worked on a finite element formulation using a weighted residual method. In another study, Bahram et al [4] investigated the vibration analysis of circular plates under the influence of in-plane loading resting on the Winkler foundation. The solutions were obtained using the Ritz method, it was observed that the foundation stiffness has an increasing influence on the natural frequencies. Also, critical buckling load was increased for the laminated circular plate. On the application of the semi-analytical 


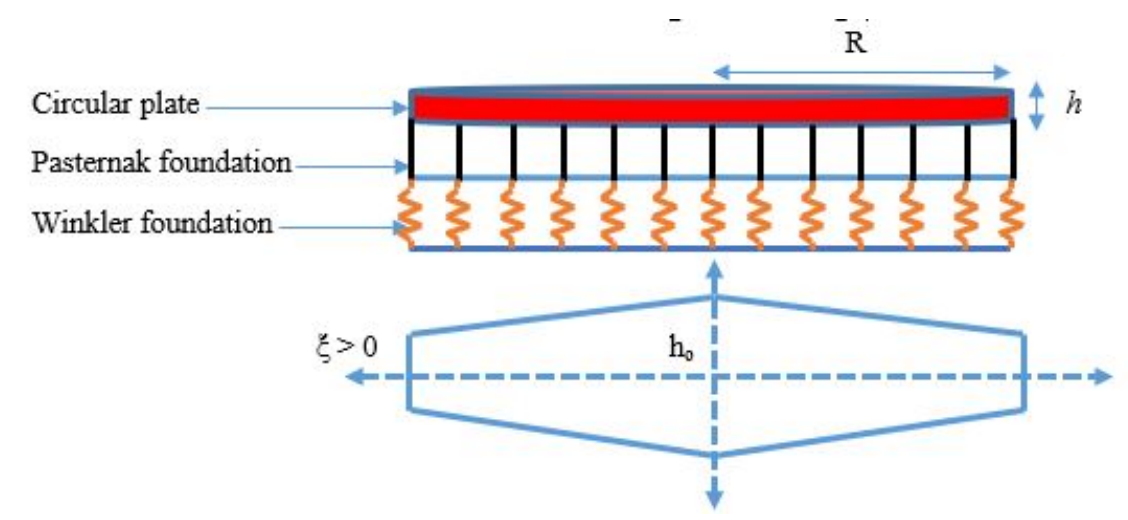

FiguRE 1. Varying thickness circular plate resting on two-parameter foundations.

method, Wei-Ming et al. [5 proposed a new semi-analytical method for obtaining Eigenvalues of vibrating circular plates with several holes, the influence of the holes on the natural frequencies was determined.

Previous studies show that the inherent singularity issue of a circular plate is not easy to handle. The numerical method is a reliable method of solution for handling the governing equations of related challenges but, the convergence, volume of iterations and stability studies associated with numerical increases the computation time and cost. Meanwhile, an attempt to obtain a symbolic solution for the dynamic behaviour of a circular plate resting on Winkler and Pasternak foundations requires the adoption of semi-analytical and analytical methods. Yun and Temuer [6, 7, formulate an improved Homotopy perturbation method (HPM) to obtain a solution for large deflection of a simply supported circular plate. Reliable results were obtained. In a later work, Zhong and Liao 8 also used the Homotopy analysis method (HAM) to obtain the solution for the non-linear problems. In another study, Yalcin et al. 9] adopted a differential transform method (DTM) for the free vibration of a circular plate. Also, Li et al. [10] analysed a bending large deflection of a simply supported circular plate using an analytical method. The DTM is equally very versatile method, good in handling singularity and a non-trivial differential system of equations, but requires the need to manipulate the governing equation before the singularity problem is resolved and, subsequently, involve transforming the governing equation to an algebraic form. The number of iterations in the DTM is very cumbersome compared to the Galerkin method of weighted residual. Meanwhile, the HPM also suffers the setback of finding the embedded parameter and initial approximation of the governing equation that satisfies the given conditions. Nonetheless, several researches on a free vibration of circular plates using different methods have been presented in the literature [11 25]. Moreover, the reliability and flexibility of the Galerkin method of weighted residual [26] have made it more effective than any other semi-numerical method. The method is much simpler than any other approximating method of solutions. The Galerkin method of weighted residual handles the circular plate vibration problem without any manipulation of the governing equation with very precise results compared to numerical and experimental results.

The review of the past studies show that the analysis of the dynamic behaviour of varying thickness and non-homogeneous circular plates resting on Winkler and Pasternak foundations with the aid of the Galerkin method of weighted residual has not been investigated. Therefore, the present study focuses on the application of the Galerkin method of weighted residual for a dynamic analysis of a non-uniform thickness circular plate resting on Winkler and Pasternak foundations. The novelty of the present study also includes resolving the singularities problem associated with a circular plate without modifying the governing equation.

\section{Problem Formulation and mathematical analysis}

A circular plate of a varying uniform thickness and non-homogenous material resting on Winkler and Pasternak foundation in Fig. 1 is considered under various boundary conditions, simply supported, free and clamped edge conditions. According to Kirchhoff plate theory, the following assumptions are considered in the model of the governing equation.

(1.) Plate thickness is smaller compared to the dimension of the circular plate.

(2.) Normal stress is assumed negligible in the transverse direction of the circular plate.

(3.) The rotary inertia effect is negligible.

(4.) Normal to the undeformed middle surface remains straight and normal to the deformed middle surface without length stretching. 
The differential governing equation for a transverse free vibration of an isotropic circular plate with a uniform thickness as shown in Fig. 1 may be written as [12, 27, 28,

$$
\begin{aligned}
g(r) & \left\{\frac{\partial^{4} w(r, t)}{\partial r^{4}}+\frac{2}{r} \frac{\partial^{3}}{\partial r^{3}} w(r, t)-\left(\frac{2 m^{2}+1}{r^{2}}\right) \frac{\partial^{2} w(r, t)}{\partial r^{2}}+\left(\frac{2 m^{2}+1}{r^{3}}\right) \frac{\partial w(r, t)}{\partial r}-\left(\frac{4 m^{2}-m^{4}}{r^{4}}\right) w(r, t)\right\}+ \\
& \frac{\partial g(r)}{\partial r}\left\{2 \frac{\partial^{3} w(r, t)}{\partial r^{3}}+\frac{2+\nu}{r} \frac{\partial^{2} w(r, t)}{\partial r^{2}}-\frac{2 m^{2}+1}{r^{2}} \frac{\partial w(r, t)}{\partial r}+\frac{3 m^{2}}{r^{3}} w(r, t)\right\}+ \\
& \frac{\partial^{2} g(r)}{\partial r^{2}}\left\{\frac{\partial^{2} w(r, t)}{\partial r^{2}}+\nu\left(\frac{1}{r} \frac{\partial w(r, t)}{\partial r}-\frac{m^{2}}{r^{2}} w(r, t)\right)\right\}-k_{s} \frac{\partial^{2} w(r, t)}{\partial r^{2}}-k_{s} \frac{1}{r} \frac{\partial w(r, t)}{\partial r}+k_{s} \frac{m^{2}}{r^{2}} w(r, t) \\
& +k_{w} w(r, t)+\rho h \frac{\partial^{2} w(r, t)}{\partial t^{2}}=0
\end{aligned}
$$

where $r$ and $\theta$ are polar coordinates. $w$ is the transverse displacement, $\rho$ is the material density, $h$ is the thickness of the plate, flexural rigidity $D=E h^{3} / 12\left(1-\nu^{2}\right)$, Poisson's ratio is $\nu$, the elasticity coefficient is $E, K_{w}$ is the Winkler foundation and $k_{s}$ is the Pasternak foundation.

For a free vibration equation, the solution is presented in this form based on Kantorovich-type approximation

$$
w(r, t)=w(r) e^{i \omega t}
$$

Presenting the solution in a more general form, the following dimensionless parameters are used

$$
r=\frac{R}{a}, w=\frac{W}{h}, \Omega^{2}=\frac{\rho_{0} h_{0} a^{4}}{D *} \omega^{2}, K_{w}=\frac{k_{w} a^{4}}{D^{*}}, K_{s}=\frac{k_{s} a^{2}}{D^{*}} ;
$$

Applying Eq. (2) on Eq. (1), we have

$$
\begin{aligned}
g(R) & \left\{\frac{d^{4} W(R)}{d R^{4}}+\frac{2}{R} \frac{d^{3}}{d R^{3}} W(R)-\left(\frac{2 m^{2}+1}{R^{2}}\right) \frac{d^{2} W(R)}{d R^{2}}+\left(\frac{2 m^{2}+1}{R^{3}}\right) \frac{d W(R)}{d R}-\left(\frac{4 m^{2}-m^{4}}{R^{4}}\right) w(R)\right\}+ \\
& \frac{d g(R)}{d R}\left\{2 \frac{d^{3} W(R)}{d R^{3}}+\frac{2+\nu}{R} \frac{d^{2} W(R)}{d R^{2}}-\frac{2 m^{2}+1}{R^{2}} \frac{d W(R)}{d R}+\frac{3 m^{2}}{R^{3}} W(R)\right\}+ \\
& \frac{d^{2} g(R)}{d R^{2}}\left\{\frac{d^{2} W(R)}{d R^{2}}+\nu\left(\frac{1}{R} \frac{d W(R)}{d R}-\frac{m^{2}}{R^{2}} W(R)\right)\right\}-\frac{a^{2} k_{s}}{D^{*}} \frac{d^{2} W(R)}{d R^{2}}-\frac{a^{2} k_{s}}{D^{*} R} \frac{d W(R)}{d R}+\frac{a^{2} k_{s}}{D^{*}} \frac{m^{2}}{R^{2}} W(R) \\
& +\frac{a^{4} k_{w}}{D^{*}} W(R)+\frac{\rho a^{4} h}{D^{*}} \omega^{2} W(R)=0,
\end{aligned}
$$

where $\Omega$ is natural frequency respectively. while $m$ is an integer.

Linear variation of thickness is considered.

$$
h=h_{0}(1+\eta R)
$$

The thickness and flexural rigidity of the plate can be expressed as;

$$
h=h_{0} f(r), \quad D=D_{0} f^{3}(r)=D_{0} g(R),
$$

where $h_{0}$ is thickness at the center and

$$
D_{0}=\frac{E h_{0}^{3}}{12-\nu^{2}}
$$

$\eta$ and $\xi$ are the thickness and density parameters respectively.

$$
\begin{aligned}
& \left(R^{3} \eta^{3}+3 R^{2} \eta^{2}+3 \eta R+1\right)\left\{\begin{array}{l}
\frac{d^{4} W(R)}{d R^{4}}+\frac{2}{R} \frac{d^{3} W(R)}{d R^{3}}-\left(\frac{2 m^{2}+1}{R^{2}}\right) \frac{d^{2} W(R)}{d R^{2}} \\
+\left(\frac{2 m^{2}+1}{R^{3}}\right) \frac{d W(R)}{d R}-\left(\frac{4 m^{2}-m^{4}}{R^{4}}\right) W(R)
\end{array}\right\}+ \\
& \left(3 R^{2} \eta^{3}+6 R \eta^{2}+3 \eta\right)\left\{2 \frac{d^{3} W(R)}{d R^{3}}+\frac{2+\nu}{R} \frac{d^{2} W(R)}{d R^{2}}-\frac{2 m^{2}+1}{R^{2}} \frac{d W(R)}{d R}+\frac{3 m^{2}}{R^{3}} W(R)\right\}+ \\
& \left(6 R \eta^{3}+6 \eta^{2}\right)\left\{\frac{d^{2} W(R)}{d R^{2}}+\nu\left(\frac{1}{R} \frac{d W(R)}{d R}-\frac{m^{2}}{R^{2}} W(R)\right)\right\}-K_{s} \frac{d^{2} W(R)}{d R^{2}}-\frac{K_{s}}{R} \frac{d W(R)}{d R} \\
& +K_{s} \frac{m^{2}}{R^{2}} W(R)+K_{w} W(R)+(\xi R+1)(\eta R+1) \Omega^{2} W(R)=0,
\end{aligned}
$$




\subsection{BOUNDARY CONDITIONS}

The boundary conditions considered as earlier stated simply support, clamped and free edge conditions. The dimensionless form of the boundary conditions may be presented in terms of the deflection $W(R)$ as follows 8 ]

- clamped

$$
\left.W(R)\right|_{R=1}=0,\left.\quad \frac{d W}{d R}\right|_{R=1}=0,
$$

- simply supported

$$
\left.W(R)\right|_{R=1}=0,\left.\quad M_{r}\right|_{r=1}=-D\left[\frac{d^{2} W}{d R^{2}}+\nu\left(\frac{1}{R} \frac{d W}{d R}+\frac{m^{2}}{R^{2}} W\right)\right]=0,
$$

- free edge

$$
\begin{aligned}
& \left.M_{r}\right|_{r=1}=-D\left[\frac{d^{2} W}{d R^{2}}+\nu\left(\frac{1}{R} \frac{d W}{d R}+\frac{m^{2}}{R^{2}} W\right)\right]=0 \\
& \left.V_{r}\right|_{r=1}=\left[\frac{d^{3} W}{d R^{3}}+\frac{1}{R} \frac{d^{2} W}{d R^{2}}+\left(\frac{m^{2} \nu-2 m^{2}-1}{R^{2}}\right) \frac{d W}{d R}+\left(\frac{3 m^{2}-m^{2} \nu}{R^{3}}\right) W\right]=0 .
\end{aligned}
$$

The bending moment is represented as the radial shear force per unit length is represented as $V_{r}$. An nth-order differential equation requires n-number of boundary condition. Since the dimensionless Eq. (6) is a fourth-order governing equation then, four boundary conditions are expected for resolving the equation. Two of the conditions may be obtained from the external condition of the plate while the rest two are obtained from the condition at the center of the plate. The regularity conditions at the center are given as,

SYMMETRIC CASE

$$
\left.\frac{d W}{d R}\right|_{R=0}=0,\left.\quad \mathrm{~V}_{R}\right|_{R=0}=\frac{d^{3} W}{d R^{3}}=0, \quad \text { for }(m=0,2,4 \cdots)
$$

AXISYMMETRIC CASE

$$
\left.W(R)\right|_{R=0}=0,\left.\quad M_{R}\right|_{R=0}=\left.\frac{d^{2} W}{d R^{2}}\right|_{R=0}=0 \quad \text { for }(m=1,3,5 \cdots) .
$$

\section{Method of Solution: Principle of Galerkin Weighted Residual}

Galerkin method was first proposed by Walther Ritz but was credited to Soviet Engineer called Boris Galerkin [26]. The method is used for handling differential equations. The approximate solutions of the differential equations are presumed to be thoroughly approximated by a finite sum of test functions. However, the chosen method of weighted residuals are used to obtain the value of the coefficients of each resulting test function $\varphi_{i}$. The corresponding coefficients are made to reduce the error between the linear combination of test functions, and actual solution, in a chosen standard. The technique is a reliable estimated solution capable of solving a series of problems that eliminate the search for vibrational formulation. Assuming the governing equation to be

$$
L\left(\varphi_{s}(r)+g(r)\right)=0, \quad r \in \Phi .
$$

with the following boundary conditions

$$
B\left(\varphi_{s}(r), \frac{d \varphi_{s}(r)}{d r}\right)=0, \quad r \in \partial \Phi
$$

where $L$ is the linear operator, $r$ is the independent variable, $\varphi_{s}(r)$ is the unknown function, $\Phi$ is the domain, $B$ is the boundary operator and $\partial \Phi$ represents the boundary of the domain.

Residual

$$
L\left(\varphi_{s}(r)+g(r)\right)=\tilde{R}(r)(\text { Residual }) .
$$

Minimize the residual by multiplying with a weight $W$ and integrate over the domain.

$$
\int_{0}^{1} \tilde{R}(r) W(r) d r=0
$$

$W_{r}$ is the weight function, which is found through either, Galerkin of weighted residual, collocation, sub-domain, or least square method. For this study Galerkin approach is adopted. 
The approximate solution $\bar{u}(r)$ is a linear combination of trial functions which must satisfy the essential boundary condition

$$
\bar{u}(r) \approx \sum_{i=1}^{N} c_{i} \phi_{i}(r)
$$

$\phi_{i}(r)$ is the trial function, while the weight function trial function is $N$ and $i$ is the node in the domain

$$
\int_{0}^{1} \tilde{R}(r) \phi_{i}(r) d r=0 \quad \text { where } i=1,2, \ldots \ldots, N
$$

\subsection{Application of Galerkin method of Weighted Residual to the governing EQUATION}

For the sake of brevity, symmetric case regularity condition and simply supported edge condition is presented here while the same approach is used to determine the other conditions treated in this study. The choice of the polynomial solution is based on the highest order of the derivative of the governing equation. This is a fourth-order derivative equation so; the chosen polynomial is of the order five.

Assume a polynomial solution for order four differential equation

$$
W(R)=a+b R+\frac{c R^{2}}{2 !}+\frac{d R^{3}}{3 !}+\frac{e R^{4}}{4 !}+\frac{g R^{5}}{5 !}
$$

Applying the boundary conditions at $R=0$, symmetric case in Eq. (10)

- Simply supported edge

$$
\begin{gathered}
W^{\prime}(0)=b=0, \\
W^{\prime \prime \prime}(0)=d=0, \\
W(1)=a+\frac{c}{2}+\frac{e}{24}+\frac{g}{120}=0, \\
-D\left[W^{\prime \prime}(1)+\nu\left(\frac{1}{R} W^{\prime}(1)+\frac{m^{2}}{R^{2}} W(1)\right)\right] \Rightarrow-\frac{221793 c}{1000}-\frac{187671 e}{2000}-\frac{244541 f}{8000}=0,
\end{gathered}
$$

For the symmetric case $m=0$ and asymmetric case $m=1$. Solving the simultaneous equation [Eqs. 20] and [21] to find the unknowns and substitute back to Eq. 17.

$$
W \Rightarrow a+\frac{1}{2} c R^{2}+\frac{1}{24}\left(-\frac{5160 a}{83}-\frac{2268 c}{83}\right) R^{4}+\left(\frac{6360 c}{83}+\frac{15840 a}{83}\right) \frac{R^{5}}{120},
$$

Chain function $N_{i}(r)$ in Eq. (22) are $a$ and $c$, as reported in Eqs. (7f 9p) the boundary condition range is $0 \Longrightarrow 1$, invariably the integral limit for this Galerkin method is 0 to 1 .

$$
\text { Galerkin equation } \int_{0}^{1} N_{i}(R) \tilde{R} d R=0 \text {, }
$$

- Chain function

$$
\begin{gathered}
N_{1}=\frac{d W}{d a} \Rightarrow 1-\frac{215 R^{4}}{83}+\frac{132 R^{5}}{83} \\
N_{2}=\frac{d W}{d c} \Rightarrow \frac{1}{2} R^{2}-\frac{189 R^{4}}{166}+\frac{53 R^{5}}{83}
\end{gathered}
$$

Applying Eq. (23) and obtain simultaneous equations based on the chain function.

$$
\int_{0}^{1}\left(1-\frac{215 R^{4}}{83}+\frac{132 R^{5}}{83}\right)\left(\begin{array}{l}
\left(R^{3} \eta^{3}+3 R^{2} \eta^{2}+3 \eta R+1\right)\left\{\begin{array}{l}
\frac{d^{4} W}{d R^{4}}+\frac{2}{R} \frac{d^{3} W}{d R^{3}}-\left(\frac{2 m^{2}+1}{R^{2}}\right) \frac{d^{2} W}{d R^{2}} \\
+\left(\frac{2 m^{2}+1}{R^{3}}\right) \frac{d W}{d R}-\left(\frac{4 m^{2}-m^{4}}{R^{4}}\right) W
\end{array}\right\} \\
+\left(3 R^{2} \eta^{3}+6 R \eta^{2}+3 \eta\right)\left\{\begin{array}{l}
2 \frac{d^{3} W}{d R^{3}}+\frac{2+\nu}{R} \frac{d^{2} W}{d R^{2}}-\frac{2 m^{2}+1}{R^{2}} \frac{d W}{d R} \\
+\frac{3 m^{2}}{R^{3}} W
\end{array}\right\}+ \\
\left(6 R \eta^{3}+6 \eta^{2}\right)\left\{\frac{d^{2} W}{d R^{2}}+\nu\left(\frac{1}{R} \frac{d W}{d R}-\frac{m^{2}}{R^{2}} W\right)\right\}-K_{s} \frac{d^{2} W}{d R^{2}}- \\
\frac{K_{s}}{R} \frac{d W}{d R}+K_{s} \frac{m^{2}}{R^{2}} W+K_{w} W+(\xi R+1)(\eta R+1) \Omega^{2} W
\end{array}\right) d R=0,
$$


Validating the analytical solutions requires assign values for the thickness parameter.

Solution of Eq. 26) becomes:

$$
\begin{aligned}
& -\frac{104333 c}{8709}-\frac{-21153 a}{1168}+\frac{2114 \Omega^{2} c \xi}{33391}+\frac{30621 a K_{s}}{33884}+\frac{7544 c K_{w}}{226609}+\frac{7544 \Omega^{2} c}{226609}+\frac{38892 c K_{s}}{79283}+ \\
& +\frac{19625 a K_{w}}{30401}+\frac{19625 \Omega^{2} a}{30401}=0 \\
& \int_{0}^{1}\left(\frac{1}{2} R^{2}-\frac{189 R^{4}}{166}+\frac{53 R^{5}}{83}\right)\left(\begin{array}{c}
\left.\left(R^{3} \eta^{3}+3 R^{2} \eta^{2}+3 \eta R+1\right)\left\{\begin{array}{c}
\frac{d^{4} W}{d R^{4}}+\frac{2}{R} \frac{d^{3} W}{d R^{3}}-\left(\frac{2 m^{2}+1}{R^{2}}\right) \frac{d^{2} W}{d R^{2}} \\
+\left(\frac{2 m^{2}+1}{R^{3}}\right) \frac{d W}{d R}-\left(\frac{4 m^{2}-m^{4}}{R^{4}}\right) W
\end{array}\right\}\right)\left\{\begin{array}{c}
2 \frac{d^{3} W}{d R^{3}}+\frac{2+\nu}{R} \frac{d^{2} W}{d R^{2}}-\frac{2 m^{2}+1}{R^{2}} \frac{d W}{d R} \\
+\frac{3 m^{3}}{R^{3}} W \\
+\left(3 R^{2} \eta^{3}+6 R \eta^{2}+3 \eta\right) \\
\left(6 R \eta^{3}+6 \eta^{2}\right)\left\{\frac{d^{2} W}{d R^{2}}+\nu\left(\frac{1}{R} \frac{d W}{d R}-\frac{m^{2}}{R^{2}} W\right)\right\}-K_{s} \frac{d^{2} W}{d R^{2}}- \\
\frac{K_{s}}{R} \frac{d W}{d R}+K_{s} \frac{m^{2}}{R^{2}} W+K_{w} W+(\xi R+1)(\eta R+1) \Omega^{2} W
\end{array}\right) d R=0 \\
W
\end{array}\right\}
\end{aligned}
$$

Solution of Eq. (28) becomes:

$$
\begin{array}{r}
\frac{21524 c}{43463}+\frac{52591 a}{27028}+\frac{3406 \Omega^{2} c \xi}{1979425}+\frac{2114 \Omega^{2} a \xi}{120859}+\frac{1939 a K_{s}}{27556}+\frac{1737 c K_{w}}{606232}+\frac{1737 \Omega^{2} c}{606232}+\frac{3617 c K_{s}}{86573}+ \\
\frac{2767 a K_{w}}{83116}+\frac{2767 \Omega^{2} a}{83116}=0
\end{array}
$$

Eq. 27) and Eq. 29) represent series expression obtained after resolving Eq. 26) and Eq. (28). The resulting simultaneous equation obtained may be written in this form

$$
\begin{aligned}
& \left(\begin{array}{l}
-\frac{104333}{8709}+\frac{2114 \Omega^{2} \xi}{120859}+\frac{7544 K_{w}}{226609} \\
+\frac{7544 \Omega^{2}}{226609}+\frac{38892 K_{s}}{79283}
\end{array}\right) c+\left(\begin{array}{l}
-\frac{21153}{1168}+\frac{7469 \Omega^{2} \xi}{33391}+\frac{30621 K_{s}}{33884} \\
+\frac{19625 K_{w}}{30401}+\frac{19625 \Omega^{2}}{30401}
\end{array}\right) a=0 \\
& \left(\begin{array}{l}
\frac{21524}{43463}+\frac{3406 \Omega^{2} \xi}{1979425}+\frac{1737 K_{w}}{606232} \\
+\frac{1737 \Omega^{2}}{606232}+\frac{3617 K_{s}}{86573}
\end{array}\right) c+\left(\begin{array}{l}
\frac{52591}{27028}+\frac{2114 \Omega^{2} \xi}{120859}+\frac{1939 K_{s}}{27556} \\
+\frac{2767 K_{w}}{83116}+\frac{2767 \Omega^{2}}{83116}
\end{array}\right) a=0
\end{aligned}
$$

The solutions are represented in terms of the natural frequency $\Omega$ and the controlling parameters. Therefore, Eq. 30 maybe written in matrix form as

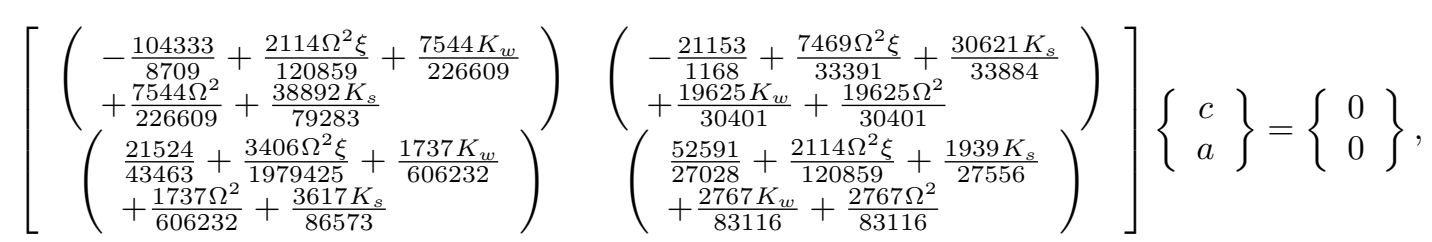

The following Characteristic determinant is obtained applying the non-trivial condition

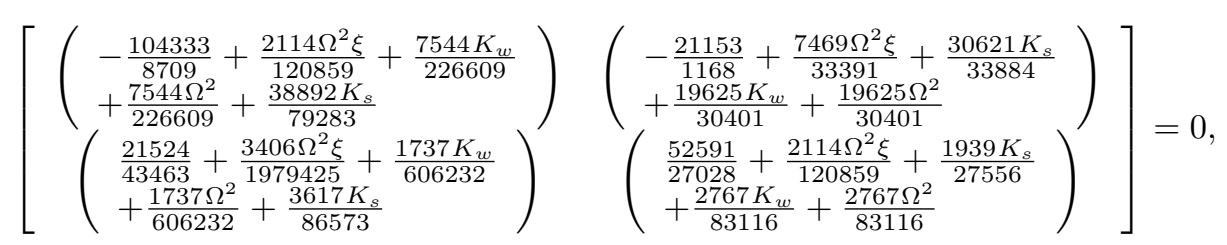

Solving Eq. (32), one gets

$$
\begin{gathered}
\left(-\frac{5975}{8059706}-\frac{72 \xi^{2}}{912077}-\frac{545 \xi}{928333}\right) \Omega^{4}+\left(\left(-\frac{857 \xi}{786779}-\frac{2995}{275111}\right) K_{s}+\left(-\frac{545 \xi}{928333}-\frac{5975}{4029853}\right) K_{w}-\right. \\
\left.\quad-\frac{17466}{29021}-\frac{21131 \xi}{82827}\right) \Omega^{2}-\frac{1074 K_{s}^{2}}{331613}+\left(\frac{15438}{36701}-\frac{2995 K_{w}}{275111}\right) K_{s}-\frac{130782}{9119}-\frac{17466 K_{w}}{29021}-\frac{5975 K_{w}{ }^{2}}{8059706}
\end{gathered}
$$


The eigenvalues are obtained resolving the Quartic equation Eq. (33) above.

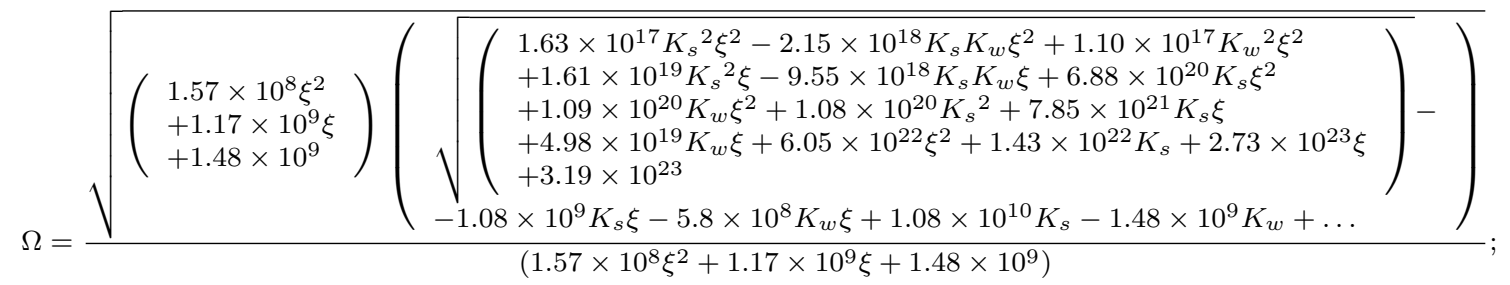

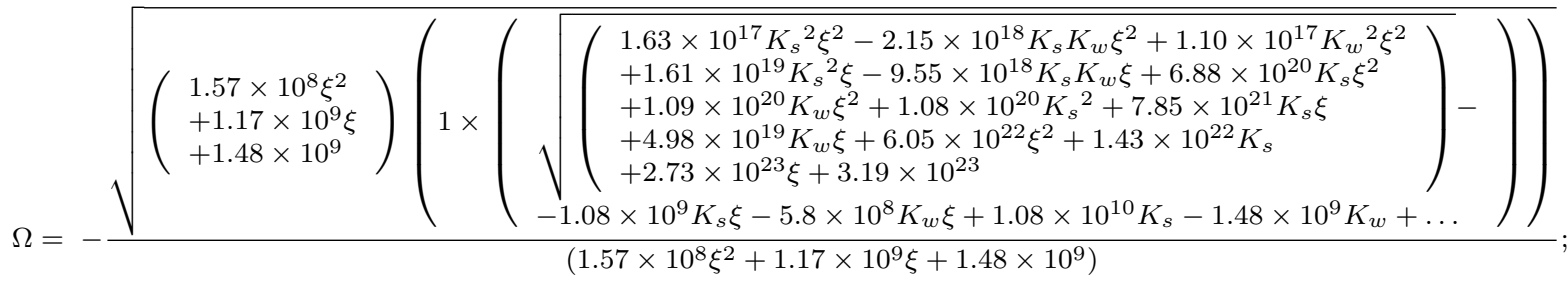

$$
\Omega=\sqrt{\left(\begin{array}{c}
1.57 \times 10^{8} \xi^{2} \\
+1.17 \times 10^{9} \xi \\
+1.48 \times 10^{9}
\end{array}\right)\left(-1 \times\left(\begin{array}{l}
\left(\begin{array}{l}
1.63 \times 10^{17} K_{s}^{2} \xi^{2}-2.15 \times 10^{18} K_{s} K_{w} \xi^{2}+1.10 \times 10^{17} K_{w}{ }^{2} \xi^{2} \\
+1.61 \times 10^{19} K_{s}^{2} \xi-9.55 \times 10^{18} K_{s} K_{w} \xi+6.88 \times 10^{20} K_{s} \xi^{2} \\
+1.09 \times 10^{20} K_{w} \xi^{2}+1.08 \times 10^{20} K_{s}^{2}+7.85 \times 10^{21} K_{s} \xi \\
+4.98 \times 10^{19} K_{w} \xi+6.05 \times 10^{22} \xi^{2}+1.43 \times 10^{22} K_{s}+2.73 \times 10^{23} \xi \\
+3.19 \times 10^{23}
\end{array}\right) \\
-1.08 \times 10^{9} K_{s} \xi-5.8 \times 10^{8} K_{w} \xi+1.08 \times 10^{10} K_{s}-1.48 \times 10^{9} K_{w}+\ldots
\end{array}\right)\right.}
$$

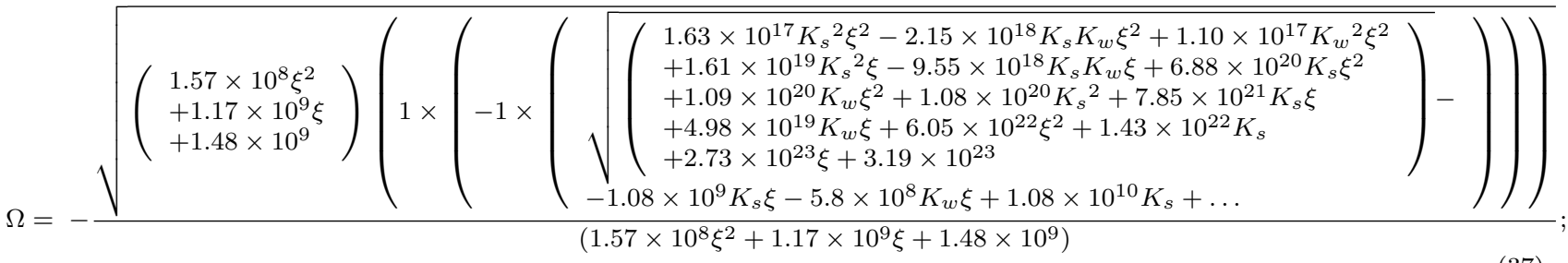

The numerical computation of the natural frequencies requires substituting values to solutions obtained in Eq. 34,37

$$
-\frac{14923 \Omega^{2}}{46030}-\frac{28704}{3259}+\frac{\sqrt{\left(4.17 \times 10^{19} \Omega^{4}+2.52 \times 10^{21} \Omega^{2}+2.52 \times 10^{22}\right)}}{2.00 \times 10^{8}},
$$

Solving the quadratic Eq. (38) gives the natural frequency.

$$
\Omega=4.957175854,-4.957175854,
$$

Substitute the positive root obtained in Eq. (39) into Eq. (31) gives,

$$
\left[\begin{array}{rr}
-\frac{137865}{4058} & -\frac{111560}{8717} \\
\frac{35062}{31091} & \frac{22757}{53569}
\end{array}\right]\left\{\begin{array}{l}
a \\
c
\end{array}\right\}=\left\{\begin{array}{l}
0 \\
0
\end{array}\right\}
$$

Putting $c=1$ in Eq. 40 , then $a$ is calculated as,

$$
\left\{\begin{array}{l}
a \\
c
\end{array}\right\}_{(1)}=\left\{\begin{array}{c}
-2.654608759 \\
1
\end{array}\right\}
$$

Therefore, the deflection solution of the governing Eq. (1) gives

$$
W(R)=1-\frac{25785 R^{2}}{20356}+\frac{32609 R^{4}}{93147}-\frac{9721 R^{5}}{116589},
$$

\subsection{Applichtion of Galerkin method of Weighted Residual to nOnlinear GOVERning EQUATION}

Considering a non-uniform thickness circular plate resting on nonlinear foundation in Fig. 2, Von- Kármán analogue is employed due to geometric nonlinearity condition involved. 


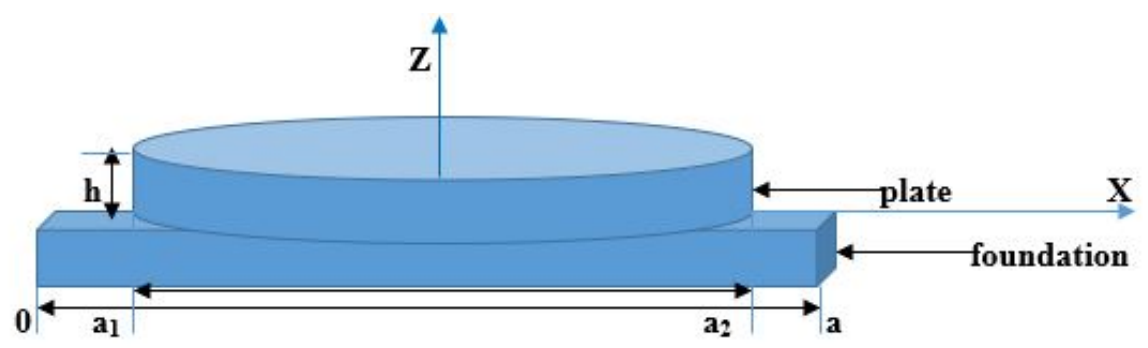

FiguRE 2. Varying thickness circular plate resting on three-parameter foundations.

$$
\begin{aligned}
& g(R)\left\{\begin{array}{l}
\frac{\partial^{4} W(R, t)}{\partial R^{4}}+\frac{2}{R} \frac{\partial^{3}}{\partial R^{3}} W(R, t)-\left(\frac{2 m^{2}+1}{R^{2}}\right) \frac{\partial^{2} W(R, t)}{\partial R^{2}}+\left(\frac{2 m^{2}+1}{R^{3}}\right) \frac{\partial W(R, t)}{\partial R}- \\
\left(\frac{4 m^{2}-m^{4}}{R^{4}}\right) w(R, t)
\end{array}\right\}+ \\
& \frac{\partial g(R)}{\partial R}\left\{2 \frac{\partial^{3} W(R, t)}{\partial R^{3}}+\frac{2+\nu}{R} \frac{\partial^{2} W(R, t)}{\partial R^{2}}-\frac{2 m^{2}+1}{R^{2}} \frac{\partial W(R, t)}{\partial R}+\frac{3 m^{2}}{R^{3}} W(R, t)\right\}+ \\
& \frac{\partial^{2} g(R)}{\partial R^{2}}\left\{\frac{\partial^{2} W(R, t)}{\partial R^{2}}+\nu\left(\frac{1}{R} \frac{\partial W(R, t)}{\partial R}-\frac{m^{2}}{R^{2}} W(R, t)\right)\right\}-\frac{a^{2} k_{s}}{D^{*}} \frac{\partial^{2} W(R, t)}{\partial R^{2}}-\frac{a^{2} k_{s}}{D^{*} R} \frac{\partial W(R, t)}{\partial R}+ \\
& \frac{a^{2} k_{s}}{D^{*}} \frac{m^{2}}{R^{2}} W(R, t)-\frac{3}{4} \frac{a^{4} k_{p}}{D^{*}}(W(R, t))^{3}+\frac{a^{4} k_{w}}{D^{*}} W(R, t)+\frac{\rho a^{4} h}{D^{*}} \frac{\partial^{2} W(R, t)}{\partial t^{2}}-\frac{1}{R} \frac{\partial}{\partial R}\left(\varphi \frac{\partial W(R, t)}{\partial R}\right)=0, \\
& \frac{\partial^{2} \varphi}{\partial R^{2}}+\frac{1}{R} \frac{\partial \varphi}{\partial R}-\frac{\varphi}{R^{2}}+\frac{E h}{2 R}\left(\frac{\partial W(R, t)}{\partial R}\right)^{2}=0
\end{aligned}
$$

Where $k_{p}$ is the nonlinear Winkler foundation and $\varphi$ is the Airy stress function.

An approximate solution is obtained by assuming the non-linear free vibrations to have the same spatial shape, i.e.,

$$
W(R, t)=\left(c_{4} R^{4}+c_{2} R^{2}+1\right) \phi(t)
$$

Substitute Eq. 45) into Eq. 444 and solve the ODE

$$
\varphi(R, t)=\frac{c_{1}}{R}-c_{3} R+\frac{(\phi(t))^{2} R^{3}\left(2 c_{4}{ }^{2} R^{4}+4 c_{2} c_{4} R^{2}+3 c_{2}{ }^{2}\right)}{6},
$$

The value of $\varphi$ is accordingly found to be finite at the origin $c_{1}=0$. Additionally, is the constant of integration is to be determined from in plane boundary conditions. Maximum value of $p h i(t)$ coincides with the maximum deflection $W_{m a} \mathrm{x}$ divided by plate thickness $h$.

The substitution of the expressions for $W$ and $\varphi$ given by Eqs. (45) and (46) respectively into Eq. (44) and the application of the Galerkin procedure in the nonlinear time differential equation obtained in the form

$$
\begin{gathered}
\int_{0}^{1} L^{\prime}(W, \varphi) W R d R=0 \\
L^{\prime}(W, \varphi)=\left(R^{3} \eta^{3}+3 R^{2} \eta^{2}+3 \eta R+1\right)\left(72 c_{4} \phi(t)-\frac{\left(12 c_{4} R^{2}+2 c_{2}\right) \phi(t)}{R^{2}}+\frac{\left(4 c_{4} R^{3}+2 c_{2} R\right) \phi(t)}{R^{3}}\right)+ \\
+\left(3 R^{3} \eta^{3}+6 R \eta^{2}+3 \eta\right)\left(48 c_{4} R \phi(t)+\frac{(2+\nu)\left(12 c_{4} R^{2}+2 c_{2}\right) \phi(t)}{R}-\frac{\left(4 c_{4} R^{3}+2 c_{2} R\right) \phi(t)}{R^{2}}\right)+ \\
+\left(6 R \eta^{3}+6 \eta^{2}\right)\left(\left(12 c_{4} R^{2}+2 c_{2}\right) \phi(t)+\frac{\nu\left(4 c_{4} R^{3}+2 c_{2} R\right) \phi(t)}{R}\right)+\frac{3}{4} k_{p}\left(c_{4} R^{4}+c_{2} R^{2}+1\right)^{3}(\phi(t))^{3}- \\
-k_{w}\left(c_{4} R^{4}+c_{2} R^{2}+1\right) \phi(t)-\frac{\left(R c_{3}-\frac{1}{6}(\phi(t))^{2} R^{3}\left(2 c_{4}{ }^{2} R^{4}+4 c_{2} c_{4} R^{2}+3 c_{2}^{2}\right)\right)\left(12 c_{4} R^{2}+2 c_{2}\right) \phi(t)}{R}- \\
-\frac{\left(c_{3}-\frac{1}{2}(\phi(t))^{2} R^{2}\left(2 c_{4}{ }^{2} R^{4}+4 c_{2} c_{4} R^{2}+3 c_{2}^{2}\right)-\frac{1}{6}(\phi(t))^{2} R^{3}\left(8 c_{4}^{2} R^{3}+8 c_{2} c_{4} R\right)\right)\left(4 c_{4} R^{3}+2 c_{2} R\right) \phi(t)}{r}+ \\
+k_{s}\left(12 c_{4} R^{2}+2 c_{2}\right) \phi(t)+\frac{k_{s}\left(4 c_{4} R^{3}+2 c_{2} R\right) \phi(t)}{R}-(\xi R+1)(\eta R+1)\left(c_{4} R^{4}+c_{2} R^{2}+1\right) \frac{\mathrm{d}^{2}}{\mathrm{~d} t^{2}} \phi(t), \quad(48)
\end{gathered}
$$


We have

$$
[M]\left\{\frac{d^{2} \ddot{\phi}_{s}}{d t^{2}}\right\}+[k]\left\{\phi_{s}\right\}-[V]\left\{\phi_{s}^{3}\right\}=\{0\},
$$

where

$$
\begin{aligned}
& M=\frac{\left(((-11088 \xi-12320) \eta-12320 \xi-13860) c_{2}+(-13860 \xi-15840) \eta-15840 \xi-18480\right) c_{4}}{55440}+ \\
& +\frac{((-6930 \xi-7920) \eta-7920 \xi-9240) c_{2}^{2}}{55440}+\frac{((-18480 \xi-22176) \eta-22176 \xi-27720) c_{2}}{55440}+ \\
& +\frac{((-4620 \xi-5040) \eta-5040 \xi-5544) c_{4}^{2}}{55440}+\frac{(-13860 \xi-18480) \eta}{55440}-\frac{\xi}{3}-\frac{1}{2}, \\
& K=-\frac{\left(\left(-\frac{188 \nu}{5}-\frac{3196}{15}\right) \eta^{3}+(-72 \nu-504) \eta^{2}+\left(-\frac{216 \nu}{7}-\frac{2376}{7}\right) \eta+\frac{3 k_{w}}{5}+12 c_{3}-12 k_{s}-64\right)}{6} c_{4}^{2}-
\end{aligned}
$$

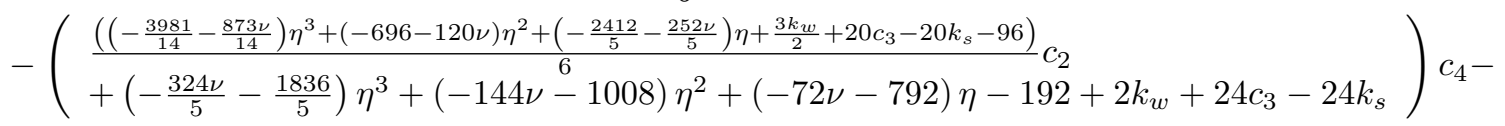

$$
\begin{aligned}
& -\frac{\left(\left(-\frac{102 \nu}{5}-\frac{102}{5}\right) \eta^{3}+(-36 \nu-36) \eta^{2}+(-12 \nu-12) \eta+6 c_{3}-6 k_{s}+k_{w}\right)}{6} c_{2}^{2}- \\
& -\frac{\left((-33 \nu-33) \eta^{3}+(-72 \nu-72) \eta^{2}+(-36 \nu-36) \eta+3 k_{w}+12 c_{3}-12 k_{s}\right)}{6} c_{2}-\frac{k_{w}}{2}, \\
& V=-\frac{\left(-\frac{k_{p}}{4}-\frac{40}{7}\right) c_{4}^{4}}{6}-\frac{\left(\left(-20-\frac{9 k_{p}}{8}\right) c_{2}-\frac{9 k_{p}}{7}-8\right) c_{4}^{3}}{6}-\frac{\left(\left(-28-\frac{27 k_{p}}{14}\right) c_{2}^{2}+\left(-20-\frac{9 k_{p}}{2}\right) c_{2}-\frac{27 k_{p}}{10}\right) c_{4}^{2}}{6}- \\
& -\frac{\left(\left(-18-\frac{3 k_{p}}{2}\right) c_{2}^{3}+\left(-20-\frac{27 k_{p}}{5}\right) c_{2}^{2}-\frac{27 c_{2} k_{p}}{4}-3 k_{p}\right)}{6} c_{4}-\frac{\left(-\frac{9 k_{p}}{20}-4\right)}{6} c_{2}^{4}-\frac{\left(-\frac{9 k_{p}}{4}-6\right)}{6} c_{2}^{3}+ \\
& +\frac{3 c_{2}^{2} k_{p}}{4}+\frac{3 c_{2} k_{p}}{4}+\frac{3 k_{p}}{8}
\end{aligned}
$$

\subsection{Application of Galerkin method of Weighted Residual to Foundation Free SURFACE}

Considering the vibration influence of foundation on the plate beyond the domain of the plate. Same procedure from Eq. 43 applies. The Galerkin procedure Eq. 47 in the nonlinear time differential equation now becomes

$$
\int_{0}^{a} L(W, \varphi) W R d R-\int_{a_{1}}^{a_{2}} L^{\prime}(W, \varphi) W R d R=0
$$

where $L(W, \varphi)$ is the foundation equation.

We have

$$
[M]\left\{\frac{d^{2} \ddot{\phi}_{s}}{d t^{2}}\right\}+[k]\left\{\phi_{s}\right\}-[V]\left\{\phi_{s}^{3}\right\}=\{0\},
$$

where

$$
\begin{aligned}
& M=\frac{c_{4}^{2}}{55440}\left(-4620 \xi \eta a_{1}{ }^{12}+(-5040 \xi-5040 \eta) a_{1}^{11}-5544 a_{1}^{10}+4620 a_{2}{ }^{10}\left(\begin{array}{c}
\frac{6}{5}+\xi \eta a_{2}{ }^{2}+ \\
\left(\frac{12 \xi}{11}+\frac{12 \eta}{11}\right) a_{2}
\end{array}\right)\right)+
\end{aligned}
$$

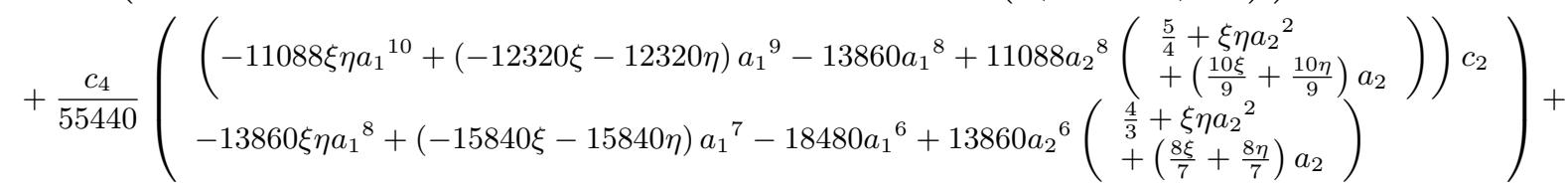

$$
\begin{aligned}
& +\frac{c_{2}{ }^{2}}{55440}\left(-6930 \xi \eta a_{1}{ }^{8}+(-7920 \xi-7920 \eta) a_{1}{ }^{7}-9240 a_{1}{ }^{6}+6930 a_{2}{ }^{6}\left(\frac{4}{3}+\xi \eta a_{2}{ }^{2}+\left(\frac{8 \xi}{7}+\frac{8 \eta}{7}\right) a_{2}\right)\right)+ \\
& +\frac{\left(-18480 \xi \eta a_{1}{ }^{6}+(-22176 \xi-22176 \eta) a_{1}{ }^{5}-27720 a_{1}{ }^{4}+18480 a_{2}{ }^{4}\left(\frac{3}{2}+\xi \eta a_{2}{ }^{2}+\left(\frac{6}{5} \xi+\frac{6}{5} \eta\right) a_{2}\right)\right) c_{2}}{55440}- \\
& -\frac{1}{4} a_{1}{ }^{4} \eta \xi+\frac{(-18480 \xi-18480 \eta) a_{1}^{3}}{55440}-\frac{1}{2}{a_{1}}^{2}+\frac{1}{4}\left(\xi \eta a_{2}{ }^{2}+2+\left(\frac{4}{3} \xi+\frac{4}{3} \eta\right) a_{2}\right) a_{2}{ }^{2},
\end{aligned}
$$




$$
\begin{aligned}
& K=\frac{1}{24}\left(\begin{array}{l}
\left(\left(\frac{432 \nu}{5}+\frac{2448}{5}\right) \eta^{3}-\frac{12 k_{w}}{5}\right) a_{1}{ }^{10}+64 \eta^{3}\left(\nu+\frac{17}{3}\right) a_{1}{ }^{9}+\left((288 \nu+2016) \eta^{2}+48 k_{s}-48 c_{3}\right) a_{1}{ }^{8} \\
+\frac{864 \eta(11+\nu) a_{1}}{7}+256 a_{1}{ }^{6}+\left(\left(-\frac{432 \nu}{5}-\frac{2448}{5}\right) \eta^{3}+\frac{12 k_{w}}{5}\right) a_{2}{ }^{10}-64 \eta^{3}\left(\nu+\frac{17}{3}\right) a_{2}{ }^{9} \\
+\left((-288 \nu-2016) \eta^{2}-48 k_{s}+48 c_{3}\right) a_{2}{ }^{8}-\frac{864 \eta(11+\nu) a_{2}{ }^{7}}{7}-256 a_{2}{ }^{6}-\frac{12 a^{8}\left(a^{2} k_{w}-20 k_{s}\right)}{5}
\end{array}\right) c_{4}^{2}+ \\
& +\frac{1}{24}\left((126 \nu+630) \eta^{3}-6 k_{w}\right) a_{1}^{8}+\frac{864 \eta^{3} a_{1}^{7}}{7}\left(\frac{37}{9}+\nu\right)+\left((480 \nu+2784) \eta^{2}+80 k_{s}-80 c_{3}\right) a_{1}{ }^{6}+ \\
& +\frac{1008 a_{1}^{5} \eta}{5}\left(\frac{67}{7}+\nu\right)+\cdots \\
& V=\frac{1}{24}\left(-\frac{160 a_{2}{ }^{14}}{7}+\frac{160 a_{1}{ }^{14}}{7}+k_{p} a_{1}^{18}-k_{p} a_{2}^{18}+a^{18} k_{p}\right) c_{4}{ }^{4}+\frac{1}{24}\left(\begin{array}{c}
6 a^{12} k_{p}+6 a_{1}{ }^{12} k_{p} \\
-6 a_{2}{ }^{12} k_{p}+72 a_{1}{ }^{8}-72 a_{2}{ }^{8}
\end{array}\right) c_{2}^{3}+ \\
& +\frac{1}{24}\left(\begin{array}{l}
\left(80 a_{1}{ }^{12}-80 a_{2}{ }^{12}-\frac{9}{2} k_{p} a_{2}{ }^{16}+\frac{9}{2} k_{p} a_{1}{ }^{16}+\frac{9}{2} a^{16} k_{p}\right) c_{2}-32 a_{2}{ }^{10}+32 a_{1}{ }^{10}+\frac{36 a_{1}{ }^{14} k_{p}}{7} \\
-\frac{36 a_{2}{ }^{14} k_{p}}{7}+\frac{36 a^{14} k_{p}}{7}
\end{array}\right) c_{4}^{3}+ \\
& +\frac{1}{24}\left(\begin{array}{l}
\left(112 a_{1}{ }^{10}-112 a_{2}{ }^{10}+\frac{54 a^{14} k_{p}}{7}-\frac{54 a_{2}{ }^{14} k_{p}}{7}+\frac{54 a_{1}{ }^{14} k_{p}}{7}\right) c_{2}{ }^{2} \\
+\left(18 a^{12} k_{p}+18 a_{1}{ }^{12} k_{p}-18 a_{2}{ }^{12} k_{p}+80 a_{1}{ }^{8}-80 a_{2}{ }^{8}\right) c_{2} \\
+\frac{54 k_{p}\left(a^{10}+a_{1}{ }^{10}-a_{2}{ }^{10}\right)}{5}
\end{array}\right) c_{4}{ }^{2}+80 a_{1}{ }^{6}-80 a_{2}{ }^{6}+\cdots,
\end{aligned}
$$

\subsection{The STRESS-DEFLECTION EXPRESSION IS [12]}

To determine the radial and circumferential stress for the circular plate, the following dimensionless expression may be used

$$
\begin{aligned}
\sigma_{r r} & =\frac{E(r, z) z}{1-\nu^{2}}\left(\frac{d^{2} W}{d R^{2}}+\frac{\nu}{R} \frac{d W}{d R}\right), \\
\sigma_{\theta \theta} & =\frac{E(r, z) z}{1-\nu^{2}}\left(\frac{1}{R} \frac{d W}{d R}+\nu \frac{d^{2} W}{d R^{2}}\right),
\end{aligned}
$$

where $E$ is the Young modulus of the circular plate, $\nu$ is the poisons ratio and $z$ is the mid-plane of the plate.

\section{RESUlts AND DisCUSSION}

The analytical solution of the governing equation of motion of the circular plate under various boundary conditions with Galerkin method of weighted residual is hereby presented. The material properties for the thin non-uniform thickness, non-homogenous Wrought iron circular plate used are $E=200 G P a$ material density $r h o=7.70 \mathrm{~g} / \mathrm{m}^{3}$ thickness of the plate $h=0.01 \mathrm{~m}$ and Poisson's ratio $\nu=0.3$ respectively.

To validate the analytical solution of free vibration of non-homogenous and varying thickness circular plate resting on Winkler and Pasternak foundations using Galerkin method of weighted residuals, application is made to the numeric data stated above given by [9]. Galerkin method of weighted residual determined the natural frequency in dimensionless form. However, the accuracy of the analytical solutions obtained are compared with results as reported in the literature [25] and confirm in good harmony along the entire values under different boundary conditions and presented in Table 1 and 2 . Since the dimensionless value of the natural frequency $\Omega$ is obtained in the analysis, the results are valid for all thickness to radius ratio. Also, the parametric studies of the controlling factors are presented in graphical form.

The solution of the Galerkin method of weighted residual is a determinant of order of the assumed polynomial chosen. In this study, fifth-order polynomial is chosen for the fourth-order governing differential equation. The analytical solutions obtained though are limited to the first two natural frequencies but, it is good enough to predict the behaviour of the plate and the results are observed similar to results reported in the literature 27. Moreso, comparing the results obtained using Galerkin method of weighted residual to results obtained in [9] using DTM. The DTM results converged at ten iterations while the present method predict almost the same results with fewer steps of calculation using the assumed polynomial. It is also observed that to obtain a higher node of natural frequencies there is a need to increase the order of the assumed polynomial chosen at the beginning of the analysis. Results are shown in Tables 1 and 2 illustrate the fundamental natural frequencies obtained which gives a reasonable prediction of the circular plate behaviour with minimal iterations.

Tables 1 and 2 also show comparisons of results for the symmetric and asymmetric case of the present study with reported work in the literature review. There is good agreement between the present study and the results reported [27, Hence, it can be concluded that the present procedure is very effective. Fig. 3 shows comparison of linear and nonlinear vibration. It is observed that the discrepancy become significant as the vibration increases. 


\begin{tabular}{lll}
\hline \multicolumn{2}{l}{ Edge condition/Dimensionless natural frequency } & $\Omega$ \\
\hline \multirow{3}{*}{ Simply supported } & [9, 12, 27] & 4.93511 \\
& Present & 4.95717 \\
& \% Diff & 2.206 \\
\hline \multirow{3}{*}{ Clamped } & [9, 12, 27] & 10.21582 \\
& Present & 10.28571 \\
& \% Diff & 6.989 \\
\hline \multirow{3}{*}{ Free } & [9, 12, 27] & 9.00312 \\
& Present & 9.03381 \\
& \% Diff & 3.069 \\
\hline
\end{tabular}

TABLE 1. Validation of fundamental natural frequency for symmetric condition.

\begin{tabular}{lll}
\hline \multicolumn{2}{l}{ Edge condition/Dimensionless natural frequency } & $\Omega$ \\
\hline \multirow{3}{*}{ Simply supported } & [9, 27, 29] & 13.94 \\
& Present & 13.9 \\
& \% Diff & 4 \\
\hline \multirow{3}{*}{ Clamped } & {$[9,27,[29$} & 21.26 \\
& Present & 21.26 \\
& \% Diff & 0 \\
\hline \multirow{3}{*}{ Free } & 9, 27, 29] & 20.475 \\
& Present & 20.556 \\
\hline
\end{tabular}

TABLE 2. Validation of fundamental natural frequency for axisymmetric condition.

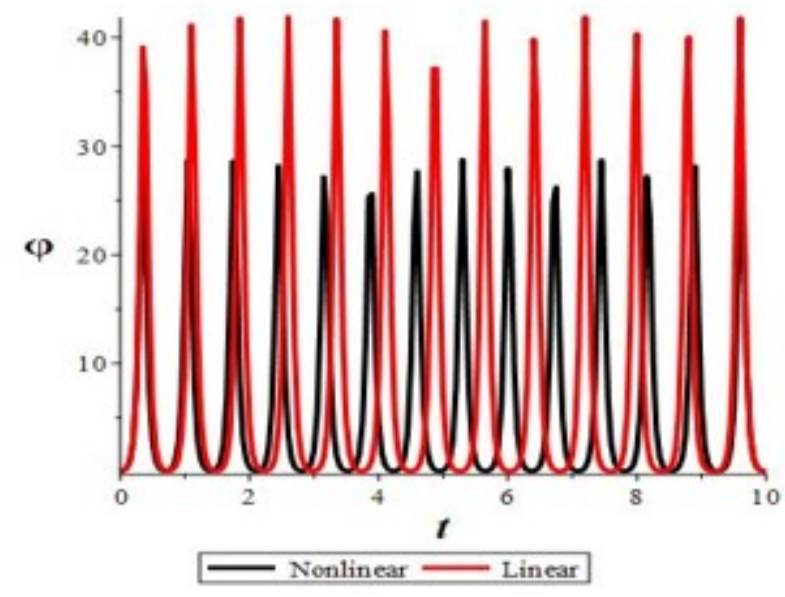

Figure 3. Comparison of dflection time history for the linear and nonlinear circular plate. 


\subsection{InVESTigAtion OF PLATE BEHAVIOUR ON FOUNDATION}

To further investigate the effect of plate resting on elastic foundation for free vibration of non-homogenous varying thickness circular plate, the natural frequencies of the solutions obtained are plotted against the foundation parameter variation. The results are obtained by setting $m=1$ and $m=0$ respectively as shown in Figs. 4 and 5 Figs. 4 and 5 show that as the elastic foundation parameter (shear stiffness) of the elastic medium increases, the natural frequency of vibration of the uniform thickness, homogenous circular plate increases. This as a result of the increased value of the elastic medium stiffness, the shear stiffness makes the uniform circular plate stronger/stiffer and vibrates at higher natural frequency. Although, it a known character of the plate to be affected by the characteristic of the elastic foundation. The same effect is also observed under the Pasternak foundation and when the plate is resting on combined Winkler and Pasternak foundations. Fig. 6 depicts the influence of varying nonlinear foundation on vibration of the circular plate, it is observed that increasing combine foundation attenuate the deflection of the circular plate. Fig. 7 illustrates the influence of foundation free surface on the vibration characteristic of the circular plate. Conversely, it is observed that as the stiffness of free foundation increases, the nonlinear vibration frequency ratio increases.

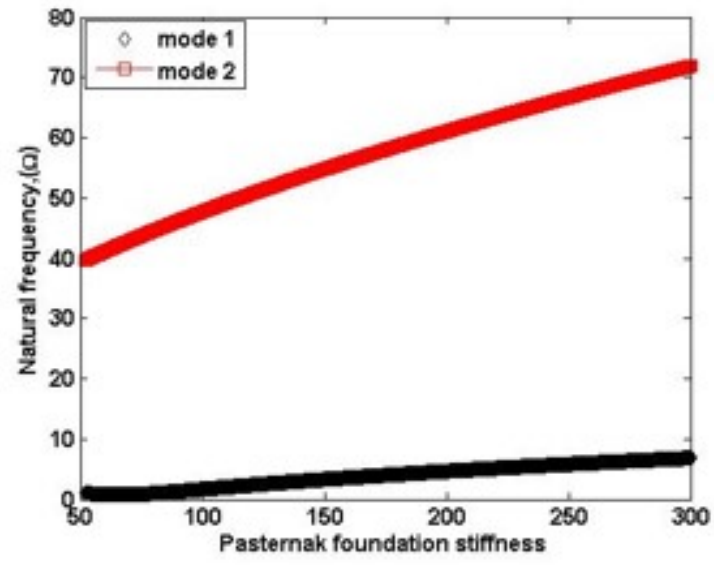

FiguRE 4. Influence of Pasternak foundation variation on symmetric and asymmetric case.

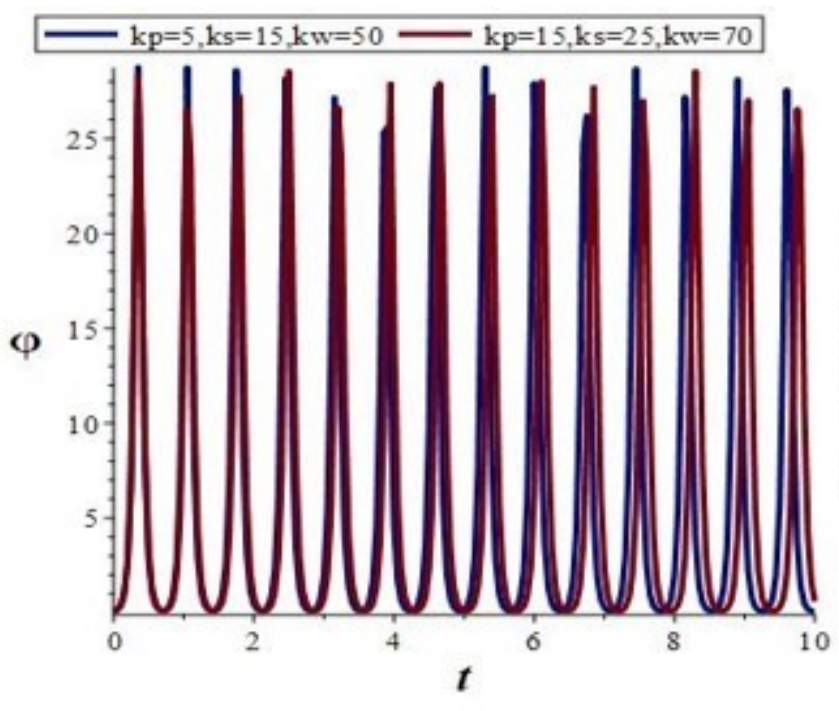

FiguRE 6. Influence of elastic foundation variation on deflection.

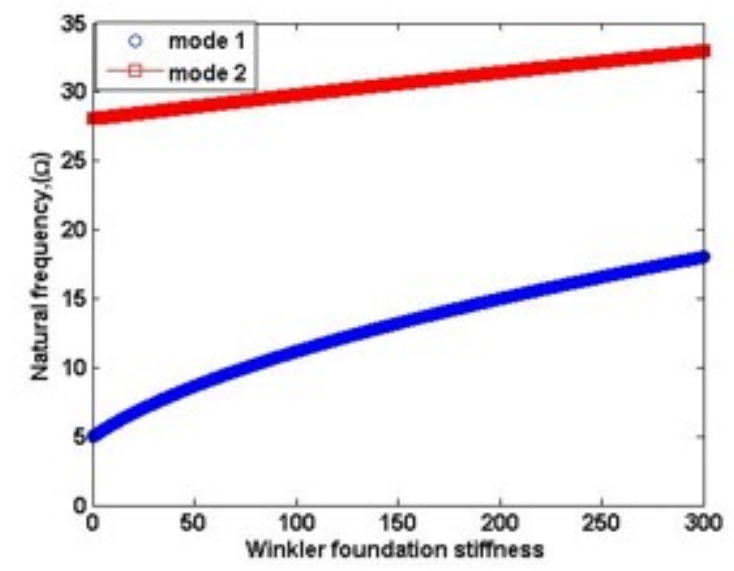

FiguRE 5. Influence of Winkler foundation variation on symmetric and asymmetric case.

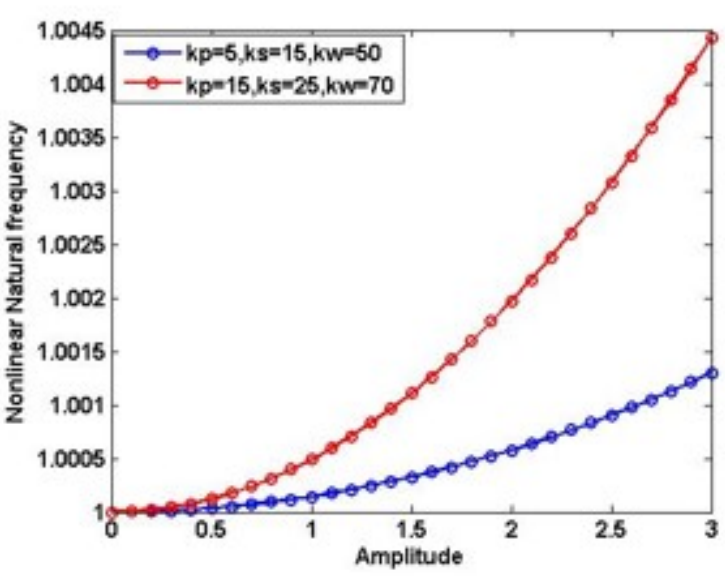

Figure 7. Influence of foundation free surface on vibration of the circular plate. 


\subsection{MODE SHAPES}

The mode shape for the dimensionless natural frequencies are shown in Figs. $8[13$ respectively. It is important to note that, the mode shape obeys the classical theory of vibration. According to [12, for transient stress investigation, the response is normally based on the modal superposition principle and the modal stress which to a certain level will expose the characteristics and content of the whole response of the plate. Based on that, the study of the non-dimension radial and circumferential stress is determined using Eq. (58) and results illustrated in Figs. 14 17. For radial and circumferential stresses, Figs. 14 17 show that the location of the vibrating node and antinodes are in-away different. The phenomenon is due to the vanishing mode of the boundary condition. it is clearly shown that, the location of the node and antinodes of the vibrating plate changes. Figs. 14,17 when compared to Figs. 813 different in the mode shape is clearly shown. Invariably, the extrema mode shapes location differs based on the boundary conditions. The extrema mode shape of the circular plate is distorted due to the presence of radial and circumferential stresses. Also comparing Figs 8 and 9 , Figs. 10 and 11 likewise Figs. 12 and 13 respectively, it is observed the symmetric case of circular plate differs from the axisymmetric case. This due to the difference in the regularity condition at the center.

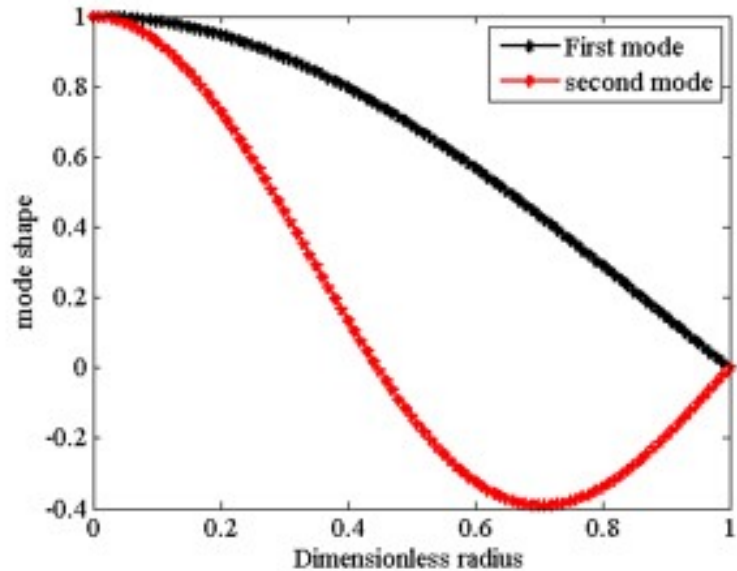

FiguRE 8. Mode shape for simply supported edge condition symmetric case.

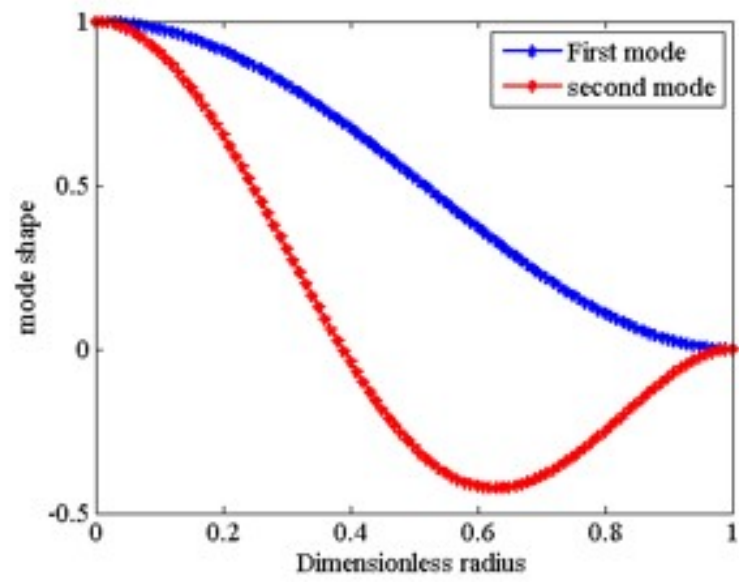

Figure 10. Mode shape for clamped edge condition symmetric case.

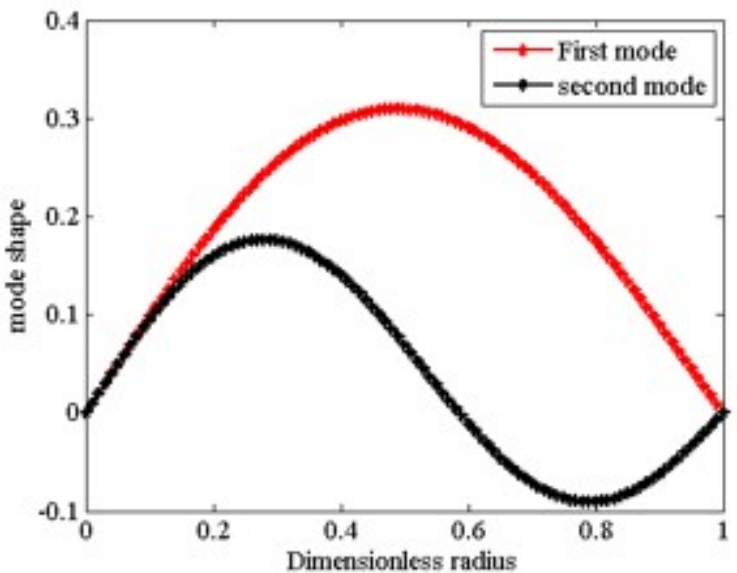

FIGURE 9. Mode shape for simply supported edge condition asymmetric case.

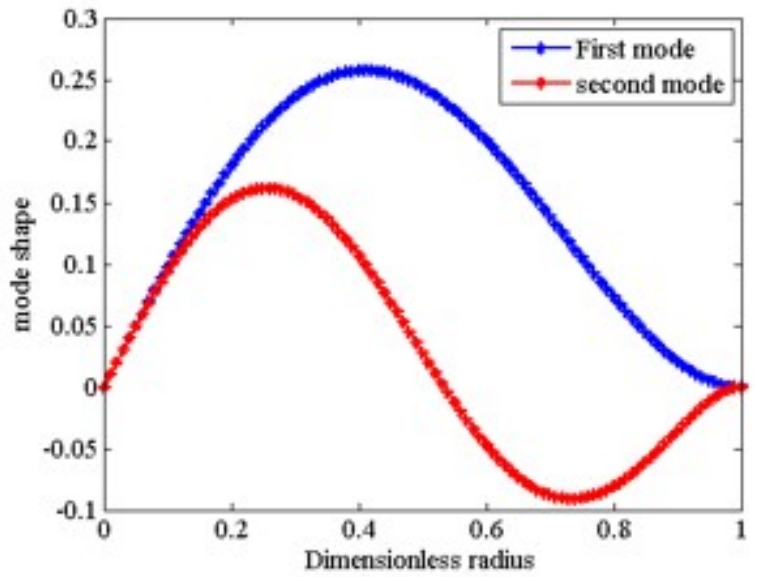

FiguRE 11. Mode shape for clamped edge condition asymmetric case. 


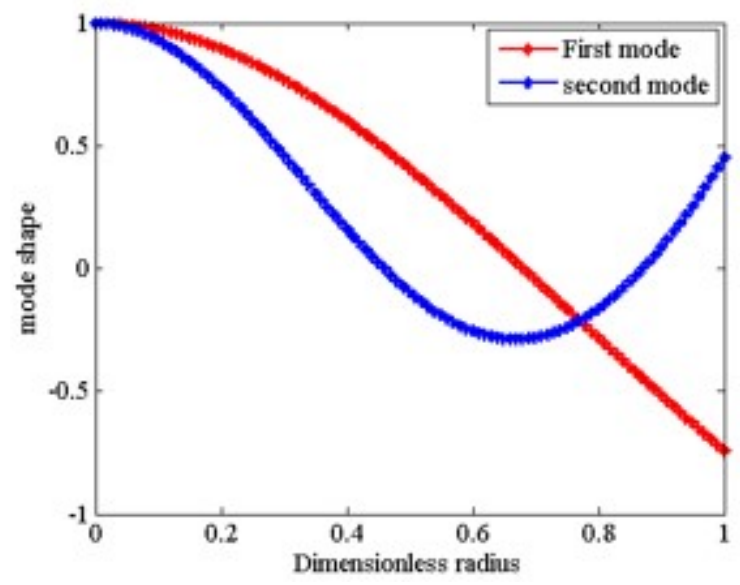

Figure 12. Mode shape for free edge condition symmetric case.

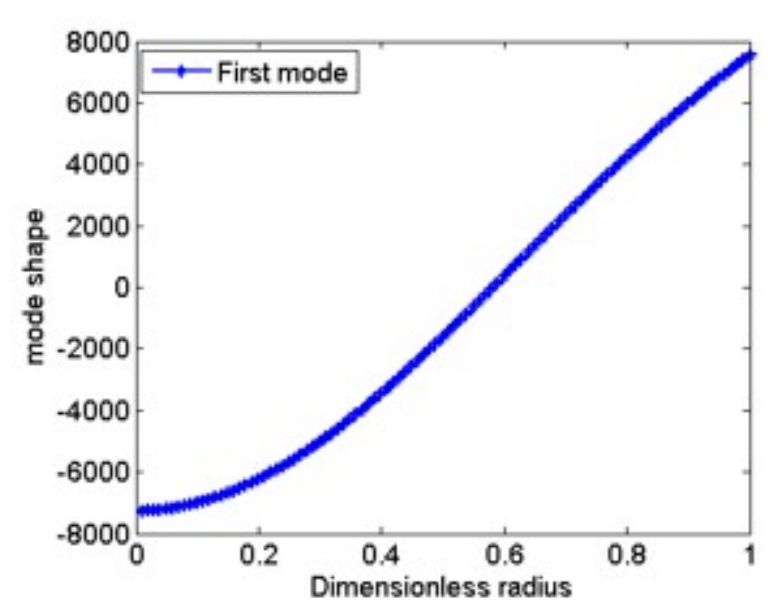

FiguRE 14. Radial stress for clamped edge symmetric case.

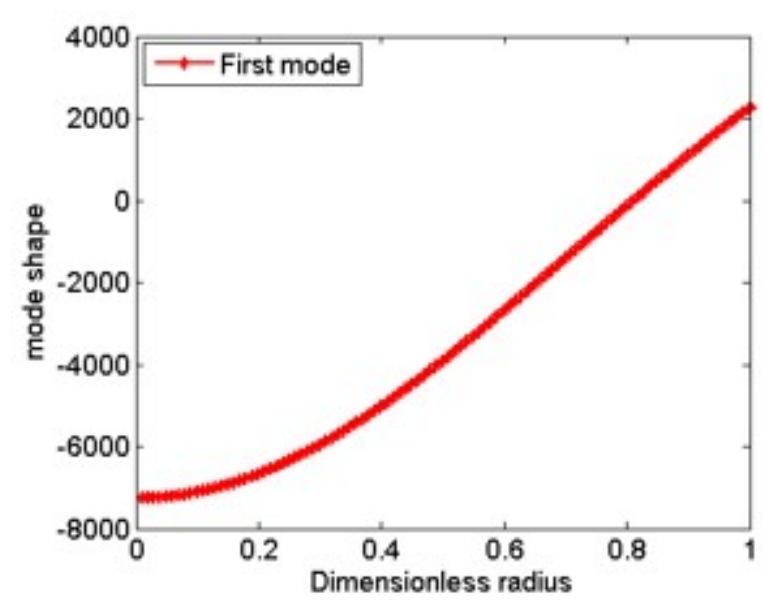

Figure 16. Circumferential stress for clamped edge symmetric case.

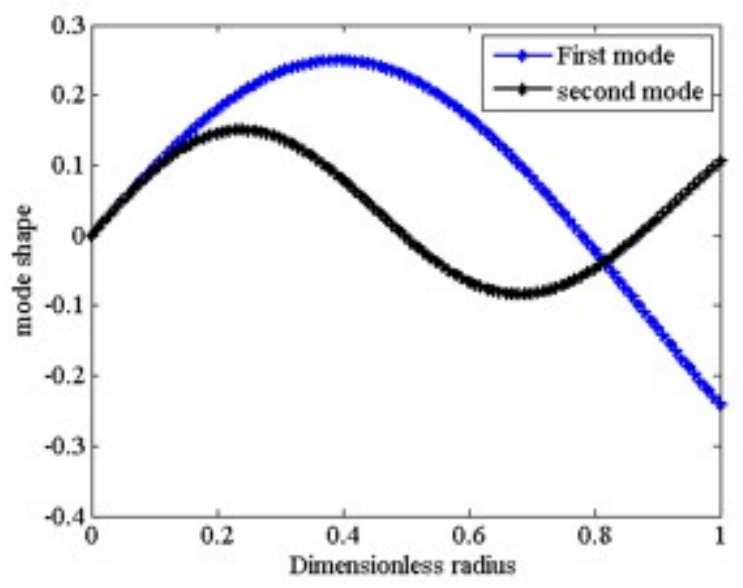

Figure 13. Mode shape for free edge condition asymmetric case.

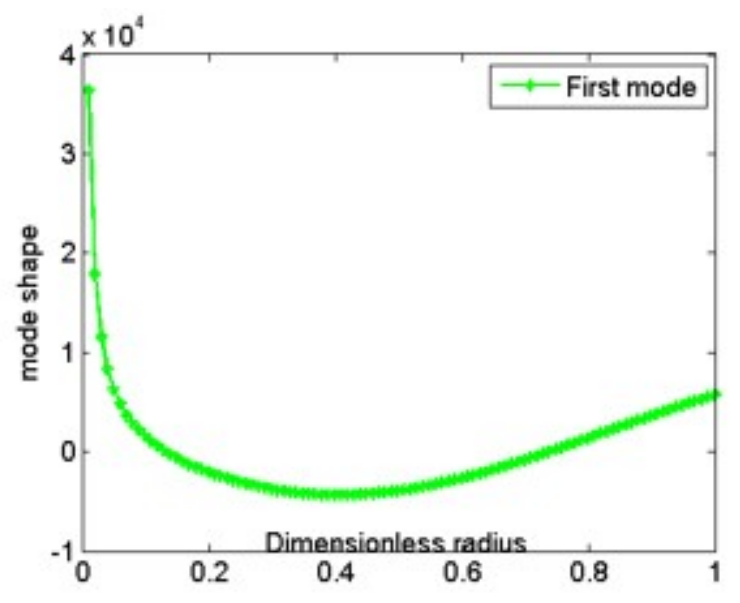

FiguRE 15. Radial stress for clamped edge asymmetric case.

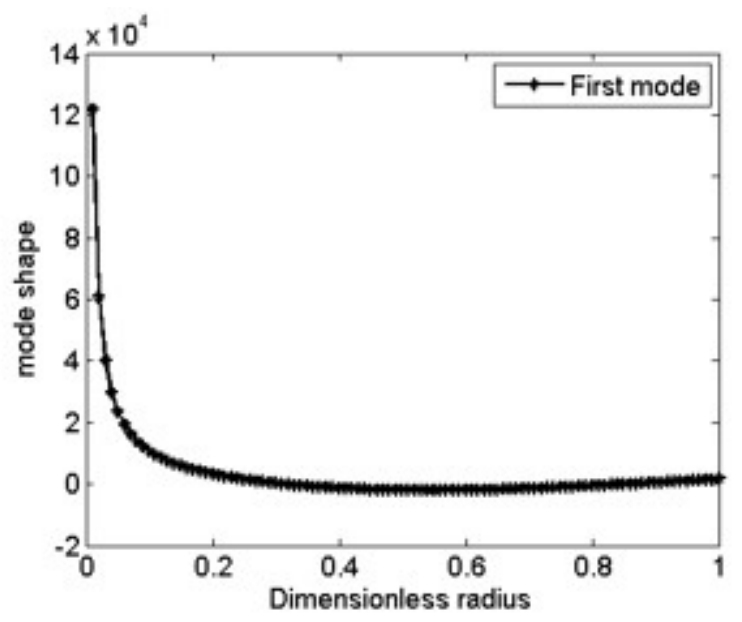

Figure 17. Circumferential stress for clamped edge asymmetric case. 


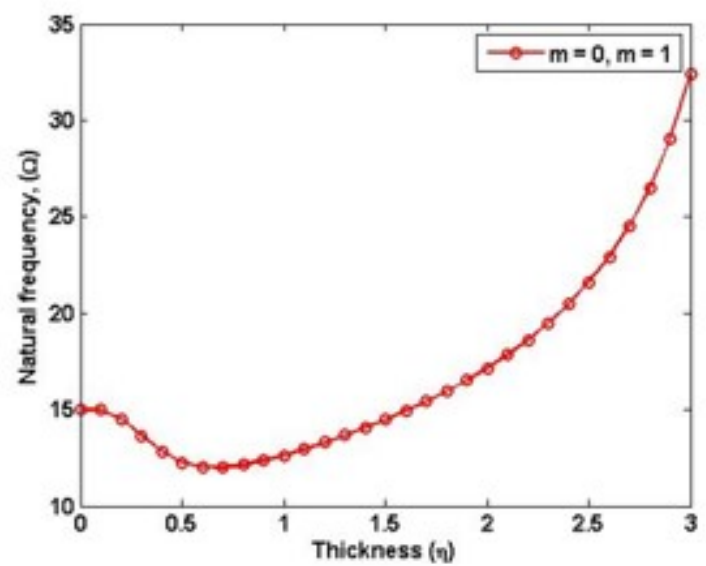

FIGURE 18. Influence of thickness parameter variation on symmetric and asymmetric case circular plate.

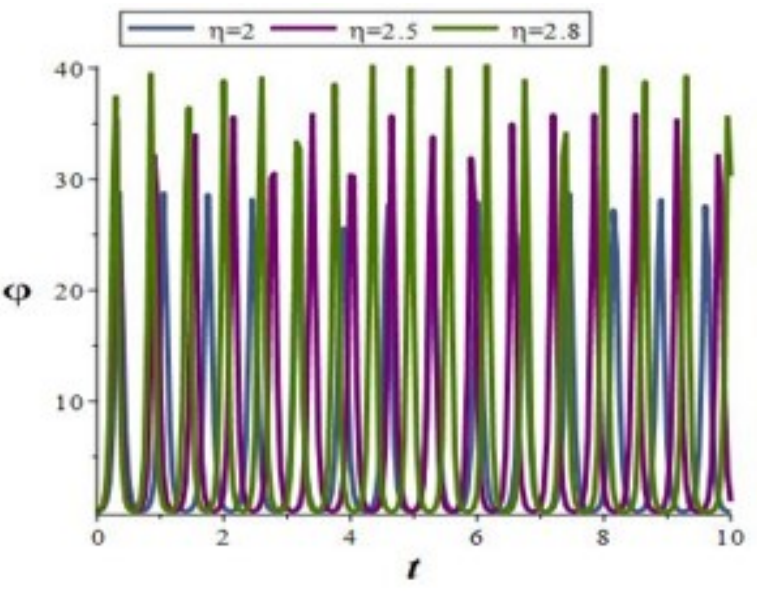

FIGURE 19. Influence of thickness parameter variation on deflection of circular plate for nonlinear foundation.

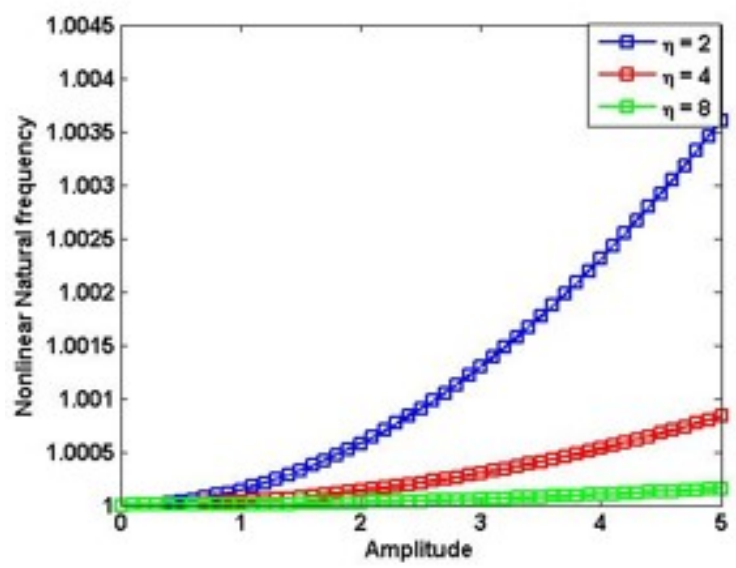

FIGURE 20. Influence of thickness parameter variation on dynamic behaviour of circular plate for free surface of foundation.

\subsection{INVESTIGATION OF PLATE THICKNESS VARIATION ON THE NATURAL FREQUENCY}

Fig. 18 illustrates the influence of the taper variation on the natural frequency. An increase in taper ratio along means that as the thickness of the plate increase, the plate gains more strength and stiffness. The more the stiffness of the plate, the more that natural frequency of the plate increase. This is an obvious result considering the classical theory of vibration. Fig. 19 shows the result obtained while investigating the influence of plate thickness variation for the circular plate resting on nonlinear foundation. From the result, it is observed that increasing the thickness, deflection of the circular plate increases. As flexibility of the plate increases, the deflection increases. Also, Fig. 20 depicts the nonlinear frequency response of the circular when the plate thickness is increase with consideration to the free surface of the foundation. A decrease in nonlinear frequency ratio is observed as the thickness increases.

\subsection{INVESTIGATION OF HOMOGENEITY PARAMETER VARIATION ON THE NATURAL FREQUENCY}

The effect of homogeneity is determined and shown in Fig. 21 Homogeneity is a function of density variation. From the results obtained, it is shown that the natural frequency decreases with an increase in density. This implies that, as the material properties are being increased, the plates gained more mass. The more the plate mass increases the more the natural frequency decreases due to the increase in the mass of the plate, as a result, the natural frequency decrease.

Fig. 22 illustrates that, increasing the homogeneity of the circular plate leads to increase in the deflection of the circular plate meanwhile, from the result illustrated in Fig. 23 increasing the density lowers the deflection of the circular plate in relation to the free surface of the foundation. 


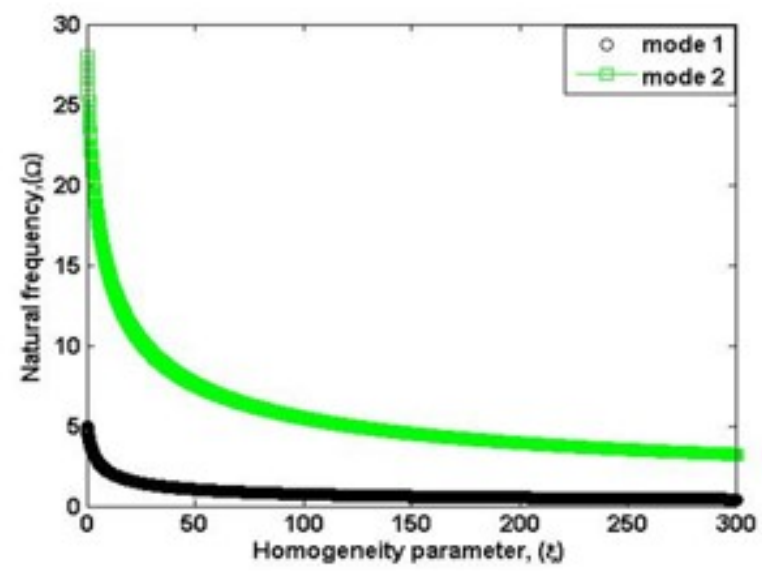

FIGURE 21. Influence of density variation on symmetric and asymmetric case circular plate.

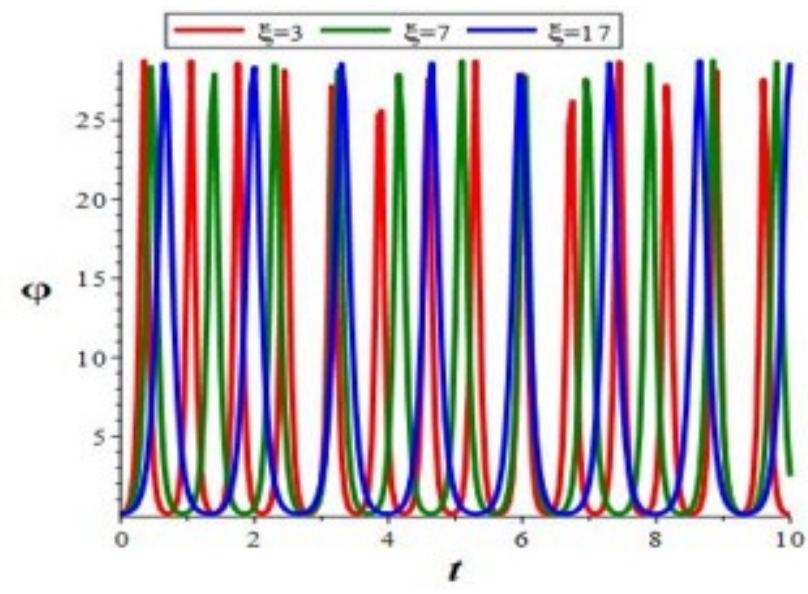

FIGURE 22. Influence of density variation on deflection of circular plate for nonlinear foundation.

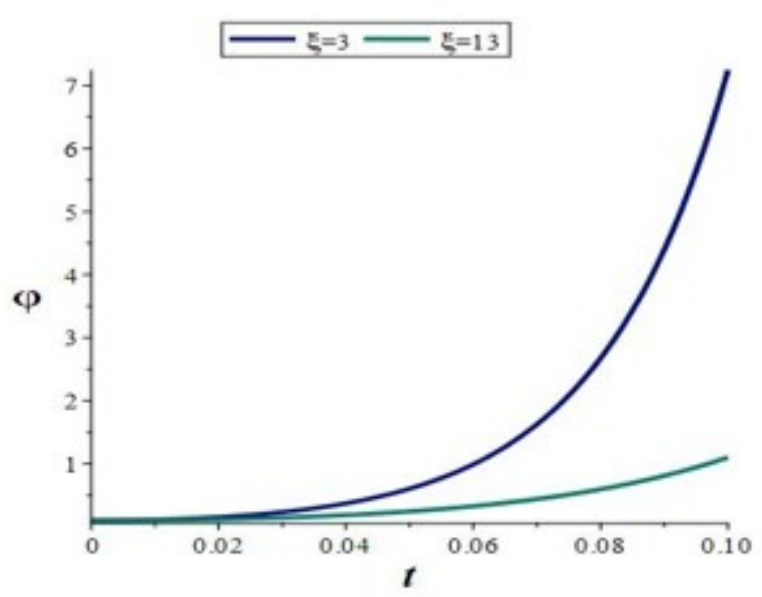

FigURE 23. Influence of density variation on deflection of circular plate for free surface of foundation.

\section{Conclusion}

In this study, free vibration of varying thickness and non-homogenous circular plate resting on Winkler and Pasternak foundations using Galerkin of weighted residual method is investigated. From the parametric studies, it was established that

(1.) The natural frequency of circular plate increases with increase in elastic Winkler foundation Parameter.

(2.) The natural frequency of circular plate increases with an increase in elastic Pasternak foundation Parameter.

(3.) Extreme modal displacement occurs on the plate due to radial and circumferential stresses.

(4.) The natural frequency of the circular plate increases with increase in the thickness of the circular plate

(5.) The natural frequency of the circular plate decreases with increase in density of the circular plate

(6.) The nonlinear vibration frequency ratio increases as the stiffness of free foundation increases. Increasing nonlinear foundation stiffness decrease the deflection of the circular plate.

(7.) Deflection of the circular plate increases as the thickness increases for nonlinear model. Conversely, the free foundation thickness increases the nonlinear frequency ratio decreases.

(8.) Increasing the homogeneity of the circular plate leads to increase in the deflection of the circular plate.

The present study emphasizes the effect of elastic foundation thickness and density variation on the dynamic behaviour of a thin circular plate. Also, the singularities issue of circular plate is handled with ease using Galerkin of weighted residual, the ease of obtaining the Eigenvalue without the difficulty of many iteration and complex mathematics is a huge benefit of the method. It is expected that the present study will contribute to the understanding of the study of the dynamic behavior of a circular plate under various parameters. 


\section{Data AVAilability}

The data used to support the findings of this study are available from the corresponding author upon request.

\section{Conflicts of interest}

The author declares that there are no conflicts of interest regarding the publication of this paper.

\section{ACKNOWLEDGEMENTS}

The author expresses sincere appreciation to the University of Lagos, Nigeria and Herzer research group, for providing material supports and a good environment for this work.

\section{LIST OF SYMBOLS \\ $r$ radius of the plate \\ $C$ clamped edge plate \\ $E$ Young's modulus \\ $F$ free edge support \\ $S$ simply supported edge \\ $\Omega$ natural frequency \\ $\frac{d}{d r} \quad$ differential operator \\ $f$ dynamic deflection \\ $h$ plate thickness \\ $\rho$ mass density \\ $D$ modulus of elasticity}

\section{REFERENCES}

[1] K.-H. Jeong, G.-M. Lee, T.-W. Kim. Free vibration analysis of a circular plate partially in contact with a liquid. Journal of Sound and Vibration 324(1):194 - 208, 2009. DOI:10.1016/j.jsv.2009.01.061

[2] M. Sari, E. Butcher. Free vibration analysis of rectangular and annular mindlin plates with undamaged and damaged boundaries by the spectral collocation method. JVC/Journal of Vibration and Control 18(11):1722 1736, 2012. DOI:10.1177/1077546311422242

[3] C. A. J. Fletcher. An improved finite element formulation derived from the method of weighted residuals. Computer Methods in Applied Mechanics and Engineering 15(2):207 - 222, 1978. DOI:10.1016/0045-7825(78)90024-5.

[4] B. Afsharmanesh, A. Ghaheri, F. Taheri-Behrooz. Buckling and vibration of laminated composite circular plate on winkler-type foundation. Steel and Composite Structures 17:1 - 19, 2014. DOI:10.12989/scs.2014.17.1.001

[5] W. Lee, J. Chen, Y. Lee. Free vibration analysis of circular plates with multiple circular holes using indirect biems. Journal of Sound and Vibration 304(3):811 - 830, 2007. DOI:10.1016/j.jsv.2007.03.026

[6] Y. shan Yun, C. Temuer. Application of the homotopy perturbation method for the large deflection problem of a circular plate. Applied Mathematical Modelling 39(3):1308 - 1316, 2015. DOI:10.1016/j.apm.2014.09.001.

[7] Y. shan Yun, C. Temuer. Application of the homotopy perturbation method for the large deflection problem of a circular plate. Applied Mathematical Modelling 39(3):1308 - 1316, 2015. DOI:10.1016/j.apm.2014.09.001.

[8] X. Zhong, S. Liao. Analytic solutions of Von Karman plate under arbitrary uniform pressure (i): Equations in differential form. Studies in Applied Mathematics 138, 2016. DOI:10.1111/sapm.12158.

[9] H. S. Yalcin, A. Arikoglu, I. Ozkol. Free vibration analysis of circular plates by differential transformation method. Applied Mathematics and Computation 212(2):377 - 386, 2009. DOI:10.1016/j.amc.2009.02.032

[10] Q. Li, J. Liu, H. Xiao. A new approach for bending analysis of thin circular plates with large deflection. International Journal of Mechanical Sciences 46(2):173 - 180, 2004. DOI:10.1016/j.ijmecsci.2004.03.012.

[11] Z. Liangchi, D. Haojiang. The method of weighted residuals for transversely isotropic axisymmetric problems and its applications to engineering. Acta Mechanica Sinica 3:261 - 267, 1987. DOI:10.1007/BF02486772.

[12] M. Shariyat. Differential transform vibration and modal stress analyses of circular plates made of two-directional functionally graded materials resting on elastic foundations. Archive of Applied Mechanics 81:1289 - 1306, 2011. DOI:10.1007/s00419-010-0484-x.

[13] D. Shi, H. Zhang, Q. Wang, S. Zha. Free and forced vibration of the moderately thick laminated composite rectangular plate on various elastic winkler and pasternak foundations. Shock and Vibration 2017, 2017.

[14] Ömer Civalek, M. H. Acar. Discrete singular convolution method for the analysis of mindlin plates on elastic foundations. International Journal of Pressure Vessels and Piping 84(9):527 - 535, 2007. DOI:10.1016/j.ijpvp.2007.07.001. 
[15] B. Akgöz, O. Civalek. Nonlinear vibration analysis of laminated plates resting on nonlinear two-parameters elastic foundations. Steel and Composite Structures 11:403 - 421, 2011. DOI:10.12989/scs.2011.11.5.403.

[16] M. Bodaghi, A. Saidi. Stability analysis of functionally graded rectangular plates under nonlinearly varying in-plane loading resting on elastic foundation. Archive of Applied Mechanics 81:765 - 780, 2011. DOI:10.1007/s00419-010-0449-0.

[17] O. Civalek, C. Demir. Buckling and bending analyses of cantilever carbon nanotubes using the euler-bernoulli beam theory based on non-local continuum model. Asian Journal of Civil Engineering 12:651 - -661, 2011.

[18] K. K. Żur. Quasi-Green's function approach to free vibration analysis of elastically supported functionally graded circular plates. Composite Structures 183:600 - 610, 2018. DOI:10.1016/j.compstruct.2017.07.012

[19] K. K. Żur. Free vibration analysis of elastically supported functionally graded annular plates via quasi-green's function method. Composites Part B: Engineering 144:37 - 55, 2018. DOI:10.1016/j.compositesb.2018.02.019

[20] K. K. Żur, P. Jankowski. Multiparametric analytical solution for the eigenvalue problem of FGM porous circular plates. Symmetry 11:429, 2019.

[21] K. K. Żur. Free vibration analysis of discrete-continuous functionally graded circular plate via the Neumann series method. Applied Mathematical Modelling 73:166 - 189, 2019. DOI:10.1016/j.apm.2019.02.047

[22] K. K. Żur. Green's function approach to frequency analysis of thin circular plates. Bulletin of the Polish Academy of Sciences Technical Sciences 64(1), 2016. DOI:10.1515/bpasts-2016-0020

[23] K. K. Żur. Green's function in frequency analysis of circular thin plates of variable thickness 53:873 - 884, 2015. DOI:10.15632/jtam-pl.53.4.873

[24] S. Salawu, G. Sobamowo. Assessment of hybrid method on investigation of dynamic behaviour of isotropic rectangular plates resting on two-parameters foundation under a creative commons attribution-NonCommercial 4.0 International License (CC BY-NC 4 13:169 - 181, 2020. DOI:10.17516/1999-494X-0213.

[25] S. Salawu, G. Sobamowo, O. Sadiq. Dynamic analysis of non-homogenous varying thickness rectangular plates resting on Pasternak and Winkler foundations. Engineering and Applied Science Letters 3:1 - 20, 2020. DOI:10.30538/psrp-easl2020.0031

[26] C. Vendhan, Y. Das. Application of Rayleigh-Ritz and Galerkin methods to non-linear vibration of plates. Journal of Sound and Vibration 39(2):147 - 157, 1975. DOI:10.1016/S0022-460X(75)80214-8.

[27] T. Wu, G. Liu. Free vibration analysis of circular plates with variable thickness by the generalized differential quadrature rule. International Journal of Solids and Structures 38(44):7967 - 7980, 2001. DOI:10.1016/S0020-7683(01)00077-4

[28] D. Zhou, S. Lo, F. Au, Y. Cheung. Three-dimensional free vibration of thick circular plates on pasternak foundation. Journal of Sound and Vibration 292(3):726 - 741, 2006. DOI:10.1016/j.jsv.2005.08.028.

[29] A. W. Leissa. Vibration of plates. Tech. rep., National Aeronautics and space administration, Washington D.C., 1970. 